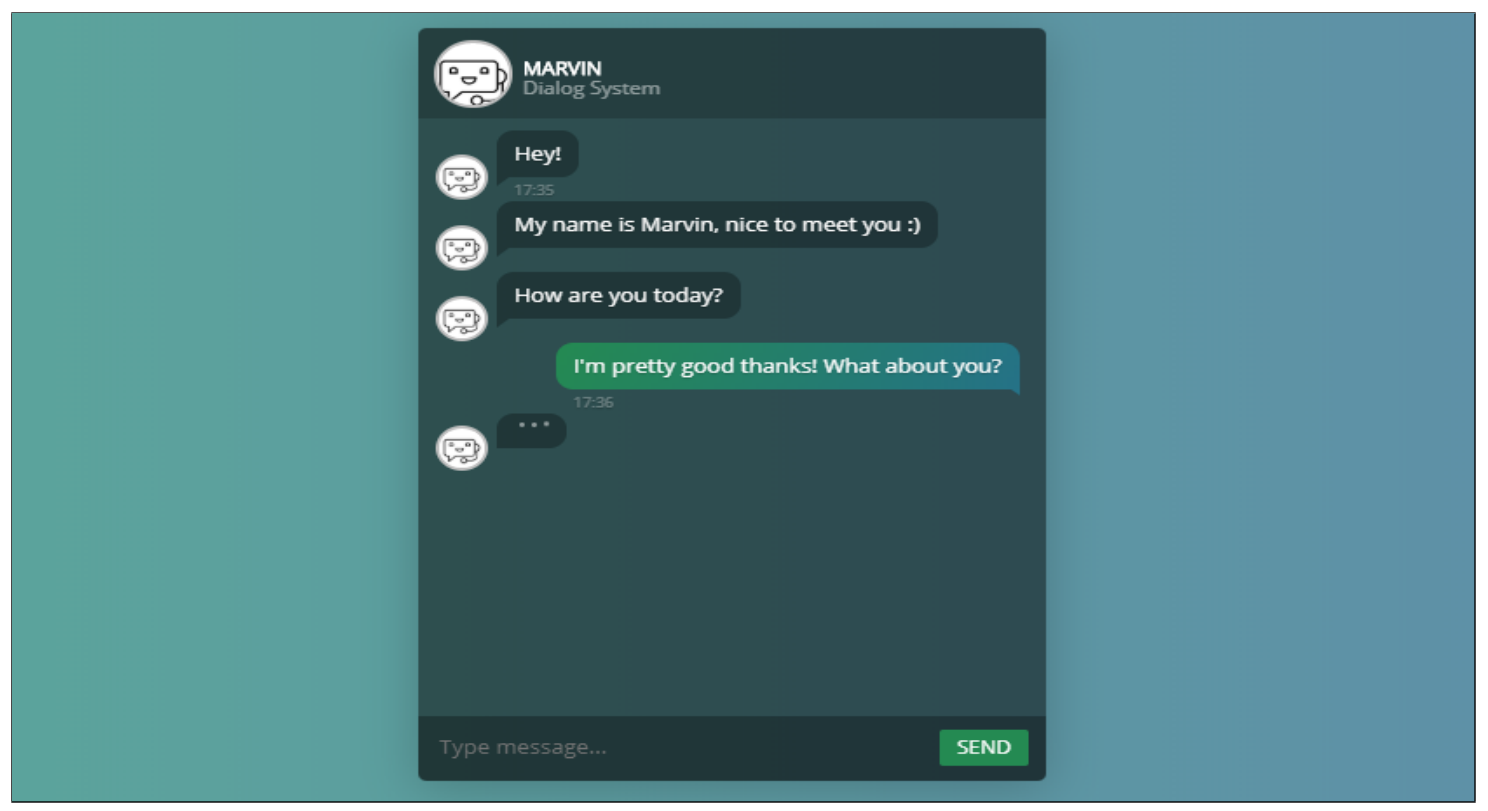

\title{
Passive Diagnosis of Mental Health Disorders Incorporating an Empathic Dialogue System
}

Through the Use of Psycholinguistics to Predict Depression and Anxiety while Incorporating Emoji Tokens in a Dialogue System to Model Empathy

Master's thesis in Applied Data Science

Fionn Delahunty 



\section{Passive Diagnosis of Mental Health Disorders Incorporating an Empathic Dialogue System}

Through the Use of Psycholinguistics to Predict Depression and Anxiety while Incorporating Emoji Tokens in a Dialogue System to Model Empathy

Fionn Delahunty

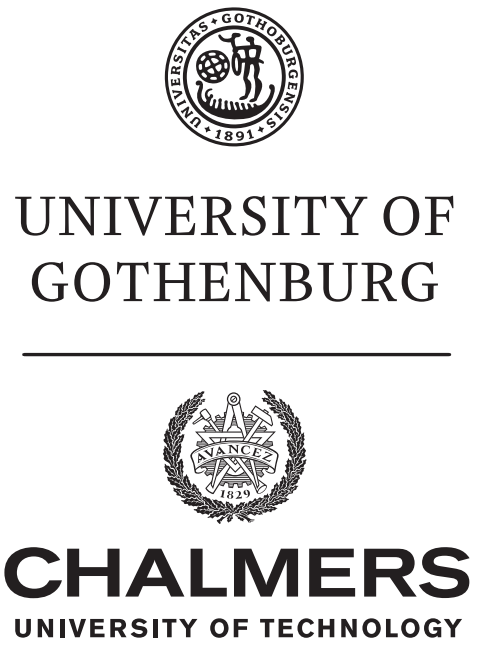

UNIVERSITY OF TECHNOLOGY

Department of Computer Science and Engineering

Chalmers University of Technology

UnIVERsity OF GothenbuRG

Gothenburg, Sweden 2019 


\section{Passive Diagnosis of Mental Health Disorders Incorporating an Empathic Dialogue System}

Through the Use of Psycholinguistics to Predict Depression and Anxiety while Incorporating Emoji Tokens in a Dialogue System to Model Empathy

Fionn Delahunty

Email: Fionnd@pm.me

(C) Fionn Delahunty, 2019.

Supervisor: Robert Johansson

Department of Computer and Information Science, Linköping University / Department of Psychology, Stockholm University, Sweden.

Advisor: Mihael Arcan

Natural Language Processing Unit, Insight Centre for Data Analytics, Data Science Institute, NUI Galway, Ireland.

Examiner: Richard Johansson

Department of Computer Science and Engineering.

Master's Thesis 2019

Department of Computer Science and Engineering

Chalmers University of Technology and University of Gothenburg

SE-412 96 Gothenburg

Telephone +4631772 1000

Cover: Interface used for the dialogue system in our experimental framework.

Typeset in $\mathrm{HT}_{\mathrm{E}} \mathrm{X}$

Gothenburg, Sweden 2019

iv 


\section{Passive Diagnosis of Mental Health Disorders Incorporating an Empathetic Dialogue System \\ Through the Use of Psycholinguistics to Predict Depression and Anxiety while Incorporating Emoji Tokens in Dialogue System to Model Empathy}

Fionn Delahunty

Department of Computer Science and Engineering

Chalmers University of Technology and University of Gothenburg

\section{Abstract}

Depression and anxiety are the two most prevalent mental health disorders worldwide, impacting the lives of millions of people each year. Current screening methods require individuals to manually complete psychometric questionnaires. In this work we develop a deep learning approach to predict psychometric scores given textual data through the use of psycholinguistics features. Data is collected via a dialogue system, were we develop and incorporate an approach to model empathy. Which aims to allow for appropriate use of these systems in a clinical setting. Following a public evaluation, we demonstrate that our approach to model empathy can out perform a similarly trained non empathic approach. Additionally, we show that our deep learning prediction approach performed well on evaluation data, but has difficulty generalizing to experimentally collected data. Limitations and implications as a result of this work are discussed.

Keywords: Clinical Psychology, Medicine, Depression, Anxiety, Psycholinguistics, Multidimensional Deep Learning, Dialogue systems, Empathy. 



\section{Acknowledgements}

A thesis is an imperfect summary of the learning and experience gain over a short, albeit concentrated period of time. This work has challenged, excited and developed me over the last eight months. I would like to my extend my utmost thanks and gratitude to both my supervisors, Robert and Mihael for their unending support and encouragement over the course of this project, both personally and academically. Additionally, to the Insight centre for supporting my work, and to all my colleagues in the NLP unit for their support and countless tea breaks.

Finally, to my friends and family. For the repeated reminder that a thesis is best done with a clear and relaxed mind.

This work has emanated from research conducted with the financial support of Science Foundation Ireland (SFI) under Grant Number SFI/12/RC/2289 (Insight).

Fionn Delahunty, Ireland, 2019 



\section{Contents}

List of Figures $\quad$ xi

List of Tables $\quad$ xiii

$\begin{array}{ll}\text { Nomenclature } & \text { Xv }\end{array}$

1 Introduction $\quad 1$

1.1 Depression and Anxiety . . . . . . . . . . . . . . . 1

1.2 Dialogue Systems . . . . . . . . . . . . . . . . . . . 2

1.3 Research Challenges . . . . . . . . . . . . . . . . 3

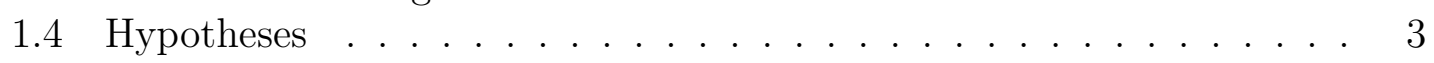

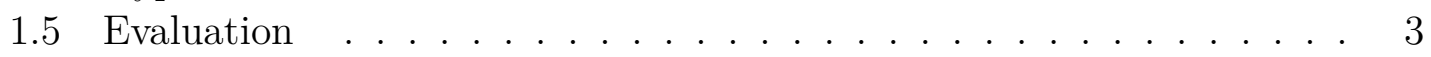

1.6 Sections Levels . . . . . . . . . . . . . . . . . 3

2 Background 5

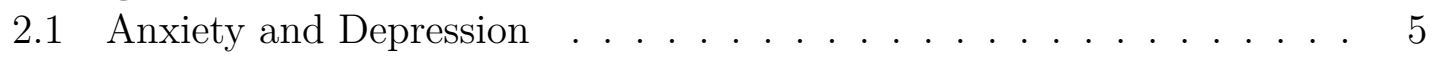

2.2 Patient Health Questionnaire . . . . . . . . . . . . . . 6

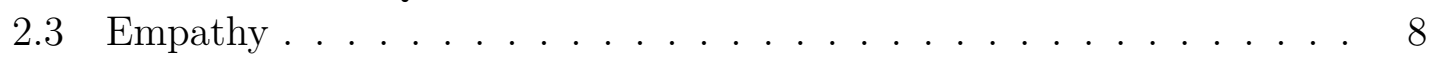

2.4 Passive Diagnosis . . . . . . . . . . . . . . . . . . . . . . . . . . . . . 9

2.5 Dialogue Algorithms . . . . . . . . . . . . . . . . . . . . . 9

2.5.1 Neural Generative Models . . . . . . . . . . . . . . . . 10

2.5.2 Long Short Term Memory . . . . . . . . . . . . . . . . . . . . 11

2.5.3 Sequence to Sequence Models . . . . . . . . . . . . . . 13

3 Related Work $\quad 15$

3.1 Machine Learning Application to Mental

Disorders . . . . . . . . . . . . . . . . 15

3.1.1 Psycholinguistics . . . . . . . . . . . . . . . . . . 15

3.1 .2 Data . . . . . . . . . . . . . . . 16

3.1 .3 Features . . . . . . . . . . . . . . . . . . . . . . . . . . . . 17

3.1 .4 Algorithms . . . . . . . . . . . . . . . . . 17

3.1 .5 Evaluation . . . . . . . . . . . . . . 18

3.2 Empathic Module . . . . . . . . . . . . . . . . 18

4 Methods 19

4.1 Machine Learning Classifier . . . . . . . . . . . . . . . . . . 19

4.1 .1 Data .............................. 19 
4.1.2 Predicting GAD-2 from the PHQ-8 . . . . . . . . . . . 20

4.1.3 Feature development . . . . . . . . . . . . . 22

4.1.3.1 Text Embedding . . . . . . . . . . . . . . . 22

4.1.3.2 Psycholinguistics . . . . . . . . . . . . . 23

4.1.3.3 Psychometric similarity . . . . . . . . . . . . 23

4.1.3.4 Feature space . . . . . . . . . . . . . . 25

4.1.4 Algorithm identification . . . . . . . . . . . . 25

4.1.5 Evolutionary tuning . . . . . . . . . . . 26

4.2 Dialogue System . . . . . . . . . . . . . . . . . . 27

4.2 .1 Data . . . . . . . . . . . . . . . . . . . 29

4.2.1.1 OpenSubtitles . . . . . . . . . . . . . . 29

4.2.1.2 DeepMoji . . . . . . . . . . . . . . . 30

4.2 .2 Dialogue Language Model . . . . . . . . . . . . . . . . . 30

4.2 .3 Implementation Pipeline . . . . . . . . . . . . . . . . 31

4.3 Evaluation system . . . . . . . . . . . . . . . . . . . . . . . . . . 32

4.4 Data Protection . . . . . . . . . . . . . . . . . 33

$\begin{array}{lll}5 & \text { Results } & 37\end{array}$

5.1 Multi-label Classifier Training . . . . . . . . . . . . . . . 37

5.1 .1 Multi-label .................. . . 37

5.1 .2 Multidimensional . . . . . . . . . . . . . . . 37

5.1 .3 Clinical Metrics . . . . . . . . . . . . . . . . 38

5.1.4 Limit of Agreement Tests . . . . . . . . . . . . . . . . . 38

5.2 Evaluation Participants . . . . . . . . . . . . . . . . . 39

$5.3 \quad H_{1}$ - Hypothesis One . . . . . . . . . . . . . . . . 39

$5.4 H_{2}$ - Hypothesis Two . . . . . . . . . . . . . . . . . . 40

6 Conclusion $\quad \mathbf{4 5}$

6.1 Overview . . . . . . . . . . . . . . . . . . . 45

6.2 Ethical Discussion . . . . . . . . . . . . . . . . . . . . . . 46

6.3 Research Questions Discussion . . . . . . . . . . . . . . . 48

6.4 Considerations and Future Work . . . . . . . . . . . . 50

Bibliography

A Appendix 1 - DeepMoji I

B Appendix 2 - Pool of Hyperparameters III

C Appendix 3 - Institutional Ethics Committee Approval V

D Appendix 4 - Participant Information Sheet VII

E Appendix 5 - Participant Distress Protocol XI

F Appendix 6 - Experimental System XIII 


\section{List of Figures}

2.1 The tripartite theory: Negative affect is shared between depression and anxiety, whereas hyperarousal is unique to anxiety and anhedonia is unique to depression. . . . . . . . . . . . . . . . . 6

2.2 A sample single layer network, with an input layer, single hidden layer, and output layer. . . . . . . . . . . . . . . . 10

2.3 A simple recurrent neural networks model, with a single hidden layer (context layer), credit from the authors of [51] . . . . . . . . . . 11

2.4 A LSTM cell with input, storage and forget gates together. . . . . . . 12

2.5 A bidirectional RNNs with two hidden layers feeding in opposite directions. Image credit from [54].

2.6 A sequence to sequence model composed of four encoders and four decoders. Source; https://github.com/farizrahman4u/seq2seq . . . . . 13

4.1 Patient Health Questionnaire (PHQ-4), four item version. . . . . . . 19

4.2 An extract of the DAIC-WOZ dataset, image credit from [47] . . . . 20

4.3 Demonstration of semantic similarity using a heat map. Sentence 1,2 and 3 are extracted from psychometric questionnaires. Sentence 4,5 are highly similar to these questions, while 6 is not. . . . . . . . . 24

4.4 Average sigmoid cross entropy score for each generation across the training . . . . . . . . . . . . . . . . . . 27

4.5 Sample annotion from the DeepMoji framework, showing the five highest probability emojis for a specific text input. . . . . . . . . 28

4.6 Visual representation of our empathic training approach. Emojis are represented by a single token of type integer_integer_integer . . . . 30

4.7 Distribution of emoji tokens across both datasets . . . . . . . . . . 31

4.8 Perplexity score across training 13 epoch iteration . . . . . . . . . . 32

4.9 Diagram of experimental protocol employed. Followed from a top down approach. . . . . . . . . . . . . . . . . 34

5.1 Confusion Matrix for predicted and true scores for each of the four PHQ-4 questions . . . . . . . . . . . . . . . . . . . 41

5.2 Responses for each of the six questions, grouped by assigned condition 42

A.1 Emoji representation in integer form . . . . . . . . . . . I

C.1 Approved ethics application from the NUI Galway institutional ethics committee. . . . . . . . . . . . . . . . . VI 
F.1 Landing page . . . . . . . . . . . . . . . XIV

F.2 Participant information page . . . . . . . . . . . XIV

F.3 Consent declaration page . . . . . . . . . . . . . XV

F.4 Instructions reminder page . . . . . . . . . . . . . . XV

F.5 Dialogue system . . . . . . . . . . . . . . . . . XVI

F.6 PHQ and empathy questionnaire . . . . . . . . . . . XVI

F.7 Finish page . . . . . . . . . . . . . . . . . X XVII 


\section{List of Tables}

4.1 Distribution of values for GAD 1 and GAD 2 questions within the dataset . . . . . . . . . . . . . . . . . . 21

4.2 Features for each GAD classification problem, features are numbered PHQ-8 questions . . . . . . . . . . . . . . . . . 22

4.3 Accuracy results of each of the four classifiers. . . . . . . . . . . . 22

4.4 Summary of aetiology theories and assessment tools . . . . . . . . . 25

4.5 Questions to evaluate the empathetic module. All questions were answered on the same three level Likert scale (Not at all, Somewhat so, Very much so) . . . . . . . . . . . . . . . . . . . . 33

5.1 Clinical Metrics for the Evaluation Data . . . . . . . . . . . . . 38

5.2 Limit of Agreements Tests for Evaluation Data . . . . . . . . . . . . 39

$5.3 \quad H_{1}$ Limit of Agreements Tests . . . . . . . . . . . . . . . . . . . 40

$5.4 \quad H_{1}$ Evaluation Metrics . . . . . . . . . . . . . . . . . 40

$5.5 H_{1}$ Clinical Metrics . . . . . . . . . . . . . . . . . . . . . 40

$5.6 \mathrm{H}_{2}$ Linear-by-Linear Association Test. ${ }^{*}=<0.05$, Given a sample size $=154, \mathrm{df}=5, \alpha=0.05$, effect size $=0.3$. We generate a power level of 0.83 and critical $x^{2}$ of $3.84 \ldots \ldots$. . . . . . . . . . . 43 


\section{Nomenclature}

\section{Medical}

Anhedonia Inability to feel pleasure in normally pleasurable activities.

Aetiology The cause, set of causes, or manner of causation of a disease or condition. 


\section{1 \\ Introduction}

Over the last decade, two simultaneous trends have emerged. The first is a more publicly recognised mental health crisis, where, according to a Lancet Commission report the worldwide "quality of mental health services is routinely worse than the quality of those for physical health" [1]. Which, the World Health Organisation (WHO) estimates results in 800,000 avoidable deaths a year [2], and many more stark statistics could be included here.

However, due to massive technological advancements in the last 20 years, we are now living in a society categorised by previously impossible to imagine levels of data collection. This is the second trend, and undoubtedly has both positive and negatives consequences, however in the last two years a new potential convergence of these trends has offered an exciting new domain.

High-Performance Medicine is proposed as the merging of artificial intelligence (AI) and medicine [3]. Resulting in a new ability to make use of innovative data to complement human decision making in hopes of saving lives. Mental health is just one sub-discipline that this new domain may offer potentially groundbreaking work. This thesis explores once such application within this area.

\subsection{Depression and Anxiety}

Some of the earliest discoveries of modern medicine date back to the time of Leonardo da Vinci, who dissected various parts of the human body and recorded the contents. Unfortunately, there is no part of the human anatomy as intrinsically complicated as the brain. Simply dissecting and viewing it provides very little information regarding its method or processes. As a result, the field of psychiatry has long struggled with classifying behaviour as normal or abnormal, and what constitutes normal functioning and disorders of the brain. To provide some consensus between the research community and front line practice the Diagnostic and Statistical Manual of Mental Disorders (DSM) which was first published in the mid 20th century, and has positioned itself as a standardised summary of the large body of research work in the field. Almost every healthcare service in the western world subscribes to the classification and symptomology contained within the DSM. It provides a standardised understanding of behavioural symptoms across countries and cultures.

The most prevalent DSM disorder is major depressive disorder ${ }^{1}$, with a lifetime prevalence rate between $15 \%$ and $17 \%$ of the population worldwide [4]. The WHO estimates 300 million people a year will suffer a depressive episode lasting a minimum

\footnotetext{
${ }^{1}$ Hereafter referred to as simply depression.
} 
of two weeks, making depression the largest cause of disability worldwide [5]. On a macro level, the disorder is characterised by a persistent period of low mood. More specifically however, the DSM requires either the presence of either persistent low mood or anhedonia to be impacting the functioning of an individual for at least two weeks before a positive diagnosis can be made.

Generalized anxiety disorder ${ }^{2}$ is the second most prevalent mental disorder worldwide, with a lifetime prevalent rate around $5 \%$ of the population [6]. The disorder is characterised by either excessive anxiety or worries about life events, often manifesting in physical symptoms such as irritability, muscle tension or sleep disturbance [7].

As discussed in the related work (Section 3) of this thesis, there is a current reliance on psychometric questionnaires for initial screening of these disorders. These questionnaires suffer from limitations such as socially desirable responding, misunderstanding and are often time consuming to complete. Recent work from the machine learning research community has looked at alternative methods to screen disorders using predictive analysis on various different data modalities $[8,9,10]$.

The patient health questionnaire (PHQ) is a short form psychometric screening questionnaire, as explained in more detail in Section 2, this is our chosen output of the psychometric classifier we develop.

\subsection{Dialogue Systems}

Dialogue systems ${ }^{3}$ are end-to-end technological systems designed to interact with users via text and they have seen successful application across various areas in the medical community over the last number of years $[11,12,13]$. These systems offer a range of advantages to the medical community, besides the ability to work in parallel with hundreds or thousands of patients at a single time, they have a low development and implement cost [14]. Additionally, they offer a high level of recording for clinical governance $[15,16]$.

Despite the potential advantages, a range of problem currently exists which limit the widespread implementation of these systems. For automatically generated dialogue content, content which is written by a data-driven algorithm as opposite to handwritten by a human, ensuring a bedside manner or awareness of empathy is vital for safe human-computer interaction $[17,18]$. A limited, but growing amount of work over the last two years has approached this nontrivial problem of developing data-driven models of empathy, which is a key requirement if these systems will ever see widespread use.

Within the mental health domain specifically, dialogue systems are already seeing widespread implementation [13], both for screening and treatment of disorders [3]. As an example, one charity in the United States (US) has processed over 109 billion text messages from US adolescents in the last six years alone and already experiments with automated data-driven replies.

Despite the limitations mentioned above, the demand for mental health support is

\footnotetext{
${ }^{2}$ Hereafter referred to as simply anxiety.

${ }^{3}$ Commonly known as chatbots.
} 
increasing and non-profit organisations often must implement automated services without full assurance that these systems are ready for real life interactions $[19,20]$.

\subsection{Research Challenges}

To refine the above areas, the research challenges we explore is how textual data can be used to predict psychometric questionnaire scores for depression and anxiety. The aim is that by using predictive analysis, we can overcome some existing limitations with the administration of these questionnaires. We do this by employing a dialogue system as a data collection method. However, to achieve this we must explore how empathy can be incorporated into these systems. Based on our literature review in Section 3, these systems have been demonstrated to remove social desirability and thus allow for more honest responding to personal questions.

\subsection{Hypotheses}

We propose two hypotheses for study within this thesis.

- $H_{1}$ - That PHQ-4 scores can be predicted from textual data using psycholinguistic features and share a significant agreement with manually completing the same questionnaire.

- $H_{2}$ - That encoding emotional values into dialogue training can provide a more empathic aware system as measured by user performance.

\subsection{Evaluation}

We propose the following approach to evaluate Hypothesis 1. We recruited participants from the public domain who had a short conversation with our proposed empathic dialogue system. Their input into the conversations were analysed and PHQ-4 values predicted using our prediction model. Participants manually complete a PHQ-4 questionnaire, and limit of agreement tests were used to examine if predicted values are similar to self-reported values.

To evaluate Hypothesis 2, we randomly allocated recruited participants to have conversations with one of two dialogue systems. One system contains the empathic module described later in this text, while the other does not. We use self-reported evaluation questions adapted from [18] to measure if participants felt one system was more empathic than the other. These questions can be found in Table 4.5.

\subsection{Sections Levels}

The contents of this thesis are laid out in five sections, following the introduction, Section 2 lays out technical knowledge in both the psychology and dialogue system domains required to fully understand the problem space. Section 3, provides a literature review of the previous work in certain areas related to this thesis and 
identification of the literature gaps we propose exploring. Section 4 which follows explains the process we employed to test our hypotheses, with the results presented in Section 5. The last section provides an overview of the whole thesis and identification of limitations and future work. 


\section{2 \\ Background}

Given the nature of a cross disciplinary thesis, in this section we provide an overview to various areas we have incorporated into the project. This should allow the reader to better understand the literature review in Section 3, and our contributions to the field outlined in sections 4 and 5 .

\subsection{Anxiety and Depression}

The fields of psychiatry and psychology concern themselves with understanding how normal human behaviour can develop into aberrant or abnormal behaviour. A range of theories have been proposed to explain how the disorders of depression and anxiety can develop in a healthy human individual. The most prominent theory is Beck's cognitive model of depression [21]. The theory is based on the psychological concept of automatic thoughts, these are though processes within our subconscious mind we are not explicitly aware of. We can generally classify these thought processes as either positive or negative. An example of a negative automatic thought might be a husband reacting with the thought "She does not respect me" when his wife did not respond to him. Beck and subsequent authors demonstrate that a variety of these thoughts processes are latent in varying degrees in all individuals [21, 22, 23].

The transition from latent state to an active state is often the result of the occurrence of a stressful life event [24]. In the case of a negative thought process becoming active, the situation is known as a negative cognitive shift. The result of this activation is that individuals begin to unconsciously filter out positive thoughts and focus on negative ones [21]. This results in the manifestation of the DSM-V symptom of "depressed mood most of the day, nearly every day".

Beck proposed that both anxiety and depression share the same negative cognitive shift. However, the trigger event in both disorders is different. In the case of anxiety, this event is often perceived by the individual to be a highly stressful life event, but objectively may not actually be. The automatic thought process here focuses more on the event itself such as catastrophizing, compared with depressed mood state where the focus is more on the long times negative outcomes of the event [21].

Over the early half of the 21st century, Beck's theory evolved into the tripartite model of anxiety and depression which was proposed by Clark and Waston [25]. As shown in Figure 2.1, this theory builds off the same concept that both disorders share a similar aaetiology in the form of negative affect. However, they differ on the manifestation of this, either developing hyperarousal or anhedonia.

There is a duality reasoning as to why this understanding of the aetiology is im- 


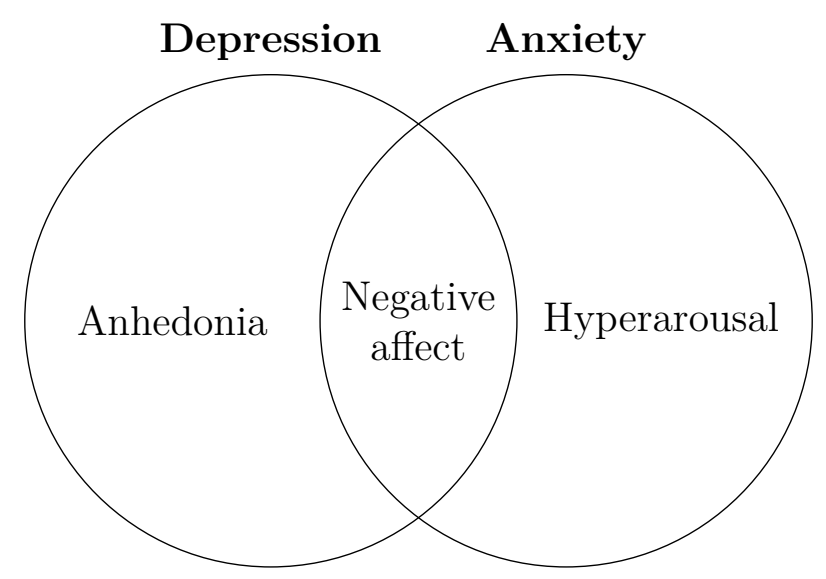

Figure 2.1: The tripartite theory: Negative affect is shared between depression and anxiety, whereas hyperarousal is unique to anxiety and anhedonia is unique to depression.

portant in the context of this thesis. Firstly, although depression and anxiety are listed as separate DSM-V disorders, clinicians will often investigate for both disorders simultaneously at the time of diagnosis. Establishing first if the negative affect is present and then which of the two unique aspects is present. Therefore, in the context of this work, it makes sense to approach the problem from a similar perspective. Secondly, an understanding of the aetiology is important to further understand why the DSM-V requires certain symptoms to be present for diagnose of depression or anxiety.

Of those existing applications of machine learning to the field of medicine, critics of these applications have argued that computer scientists and statisticians lack a true understanding of the medical aspects of the problem. Much of the existing published content comes from the perspective of a single domain, and therefore is often limited in its contributions to the overall field [3].

In Section 3 of this thesis, we will discuss a limitation of the existing work in this field with regards to how depression is always viewed as a binary occurrence. Although this makes sense from a statistical approach, it is rarely, if ever possible to fully categorise a person into such a narrow construct. Especially since many mental disorders are often considered to occur on a continuum. More recent work by the authors of [8] has looked at predicting depression on a symptomatic level instead, overcoming this limitation.

The most logical approach, in this case, is to focus on predicting the primary symptoms of each disorder. In a clinical setting, psychometric questionnaires are often employed as an initial screening tool in diagnosis. They are often short, standardised and allow for easy comparisons between patients.

\subsection{Patient Health Questionnaire}

A number of different questionnaires exist for depression and anxiety disorders, the most commonly employed type is the PHQ-4, which comes in a variety of different 
forms depending on the disorder being investigated [26]. These questionnaires maintain both high sensitivity and specificity metrics, demonstrate high validity across languages and have been successful administrated across a broad range of medical settings [26]. All questionnaires are non-commercially licensed, meaning they can be easily employed in a variety of settings.

The 4-item version which was proposed in 2009 is designed as an ultra-brief screening scale for both depression and anxiety. Based on the DSM-V classification it assesses the severity over the last two weeks of the four key symptoms for anxiety and depression. Each question is matched to one of the following symptoms, anhedonia, depressed mood, excessive anxiety and uncontrollable worry [27]. The results of the questionnaire can be interpreted in three different ways. At a most basic level, the four questions can be viewed independently on a binary cut-off (symptom presence/absent). The presence of at least one symptom could identify a disorder.

A more fine-grained view can be achieved by divide the questionnaire into two sections, composed of the two anxiety questions and the two depression questions. Combining the scores for each of the sections gives a value between $0-6$. Finally combing all scores on the questionnaire gives an overall score between $0-12$.

Despite their usefulness in many settings, psychometric questionnaires have a number of inherent limitations, one of which is social desirability impacting the honesty of responses [28]. Social desirability is the subconscious process of tailoring your behaviour in hopes of its becoming more socially acceptable [29]. In a patient-doctor setting, honesty of reporting is a vital requirement for a correct diagnosis, social desirability can result in individuals not providing full accounts to their doctors. Especially this is an issue in the case of taboo topics, such as drug use, suicide and safe sex practices $[29,30]$.

Therefore, there is a requirement to identify ways in which social desirability can be removed from the diagnosis setting. Recently, dialogue systems have been successfully applied in this domain $[11,13,30]$. This body of work demonstrates that individuals feel more comfortable discussing taboo issues when they are not required to do so in the physical presence of another individual. This can be achieved through telemedicine, wizard of oz style setups ${ }^{1}$ or fully automated dialogue systems.

Considerable work has been published over the last ten years on the how technology can be applied to the discipline of clinical psychology, from prognosis and diagnosis [31] to automated counselling sessions, and to applications of therapeutic treatment through technology [32]. These systems are often highly scalable, low cost compared to a medical professional time commitment and available on instant demand [32]. Several national healthcare providers have already implemented various forms of these systems, and a growing number of private companies exist offering various products in this space.

Within the context of this work however, our focus is how technology can remove social desirability in the patient-doctor setting. The general trend in the field of dialogue systems has been to develop increasingly human-like systems, with the famous "Turing test" as the gold standard evaluation technique. However, the authors of [33] argue that in the context of reducing social desirability, the aim should not

\footnotetext{
${ }^{1}$ Occurs when a participant interacts with a computer system that they believe to be autonomous, but is controlled by a unseen human being.
} 
be to create system that are indistinguishable from humans. Rather, imperfection is required so people are always aware they are talking to a computer.

It has been further demonstrated that individuals react psychologically to dialogue systems, even when they know they are not human [33]. This leads to individuals being less fearful of self-disclosure and displaying more intense emotions in patientcomputer conversations than would be expected. Despite the requirement that these systems maintain some characteristics of computer systems, authors of $[8,18,33,34]$ have all noted that these systems still need to maintain a certain level in empathy in all their interactions to fully be efficient in this space.

\subsection{Empathy}

Research on the construct of empathy originated from the field of medicine, generally in response to training new medical students [35]. The literature lacks a consistent definition. However, empathy is generally considered to be "the ability to detect, understand and acknowledge the emotions of another individual without directly experiencing them in oneself" [36]. Importantly, the literature consistently agrees that a key characteristic of empathy is a certain level of detachment from the emotions, which allows a clinician to maintain objectivity [36].

The importance of empathy throughout the entire medical process is repeatedly demonstrated [37, 38]. Higher levels of empathy have been shown to facilitate trust, improve disclosure and reduce anxiety between patients and doctors [36]. Many medical schools consider it vital to train students to be empathic [35]. Recent work from the field of neuropsychology has identified two biologically distinct empathic processes [39].

The primary or basic form of empathy is affective empathy, which consists of the ability to detect emotional states in others. A higher neurologically process is cognitive empathy, which is the ability to take the mental perspective of others, thus allowing inferences about their mental or emotional states [40]. A slightly alternative definition is provided by the authors of [41], where affective empathy is the ability "be sensitive to and vicariously experience the feelings of others", while cognitive empathy is "construct a working model of the emotional states of others".

Regardless, however, research has repeatably demonstrated a dual process by brain imaging and brain lesion studies in both animals and humans [42, 43]. In terms of psychopathology, it has been shown to manifest in psychiatric illnesses. Psychopaths and schizophrenics are characterised by deficits in the affective aspect, but not the cognitive aspect. While the reverse is true for autism, bipolar and borderline disorders $[39,44]$.

As we demonstrate in Section 3, much of the existing work from the field of natural language understanding has focused on affective empathy, examples of which include shared tasks were the object has been to detect certain emotional features from the text. However, very limited work has focused on cognitive empathy, where the aim is on the appropriate response in a given situation. 


\subsection{Passive Diagnosis}

As the author of [3] state, "Almost every type of clinician, ranging from specialty doctor to paramedic, will be using AI [artificial intelligence] technology, and in particular deep learning, in the future". Even within the last five years, AI, machine learning and data science have become an increasingly common tool in the medical community. These tools have supported clinicians in fields such as radiology, cardiology, oncology and psychiatry [3]. This has prompted the food and drug administration in the US to begin the process of introducing legalization where machine learning systems are involved in medical admission or decision making [45].

The authors of [3] have proposed that this combination of medicine and AI falls under the term High Performance Medicine. The work undertaken as part of this thesis also falls generally within this domain, but more specifically within a category termed Passive Diagnosis, a term was coined by the authors of [8]. In comparison to the traditional concept of active diagnosis, where an individual suffering certain symptoms would actively seek out a medical diagnosis, this process can now be facilitated by adding a passive element. Unlike a medical professional who has limited time and resources, it is feasible to have machine learning algorithms constantly passively observe an individual's health. Once these algorithms detect certain changes in an individual's health that might be indicative of a disorder, the algorithm can inform the individual and an appropriate human professional for further investigation.

This concept has been applied across both a range of medical disorders and data modalities [9, 46, 47]. Each type of modality offers potential advantages and disadvantages to completing the goal of passive diagnosis. There is an often a trade-off required between the availability and validity of data. The concept explored with Hypothesis one of this thesis is related to the this area.

\subsection{Dialogue Algorithms}

The question of designing systems to engage in human-computer interaction has long been a topical issue within the field of natural language processing. Dialogue systems (or conversational agents) are automatic computer systems intended to be able to converse with humans [48]. They can employ various modalities such as text, speech, haptics or gestures. Depending on their end-use application, these systems can either be considered to be task-oriented, focused around allowing the user to complete a certain task or non task-oriented systems focused on general nonspecific conversations $[49,50]$. Within the context of the thesis, our focus is on non-task-oriented systems.

The development of non-task-oriented systems often relies on one of two approaches, data-driven neural generative models or retrieval-based methods [50]. Both approaches must balance the generalisability of the trained model and conversational features such as fluency in responses and appropriateness. Retrieval based system employs a pipeline approach, beginning with some form of natural language understanding, an input is reduced to analysis its semantic meaning. This is then queried 


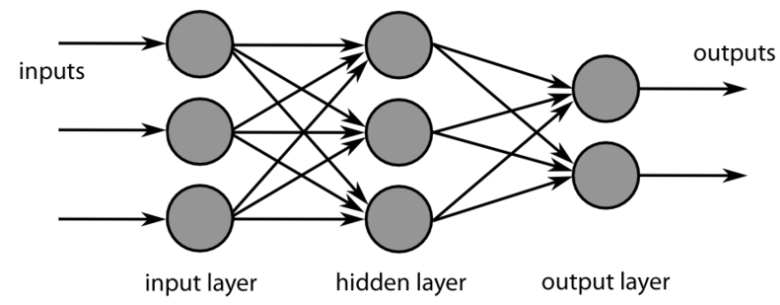

Figure 2.2: A sample single layer network, with an input layer, single hidden layer, and output layer.

against a database of prewritten possible responses. The closest matching or most appropriate response is then returned to the user.

Since all responses in these systems are prewritten by a human, these systems maintain high fluency and appropriateness provided their database contain a sufficient number of possible situations. However, expect for large systems, the generalisability of these systems can be limited, and they have no ability to scale outside of their prewritten areas. In contrast, neural generative models overcome this limitation by employing a data-driven approach in generating their own answers to inputs.

\subsubsection{Neural Generative Models}

These approaches originate from the field of statistical machine translation and are viewed as an extension of the attempt to translate a source language into a different target language. These methods work by developing a language model (LM), which is a trained probability distribution that assigns a probability likelihood to the next word in textual data given context for a sequence of previous words (Equation 2.1). Initial work demonstrated that neural networks offer high accuracy scores when used to train these LMs. Specifically, recurrent neural networks (RNNs) are applicable given their ability to model sequential data.

$$
P\left(w_{t} \mid \text { context }\right) \forall t \in V
$$

Feedforward neural networks, of which RNNs are a specific implementation are layered matrices composed of neurons and layers. At a minimum, an input layer, hidden layer and output layer are always present. The number of neurons in the input layer is equal to the number of features in the dataset. Data passes into each neuron on the input layer, if the neuron allows, the data will pass to the subsequent neuron in the next layer in a forward moving fashion.

For each neuron in an feed forward neural network (FNNs), it takes an input value $x$ which is then multiplied by a weight value $w$. If the neuron accepts multiple inputs, each input follows the same process. All combined inputs and weights are then added to a bias term $b$. The sum of this equation is then compared against a threshold level (activation function $f$ ), to determine if the new value passes into the next neuron in the feed.

$$
f(x * w+b)
$$




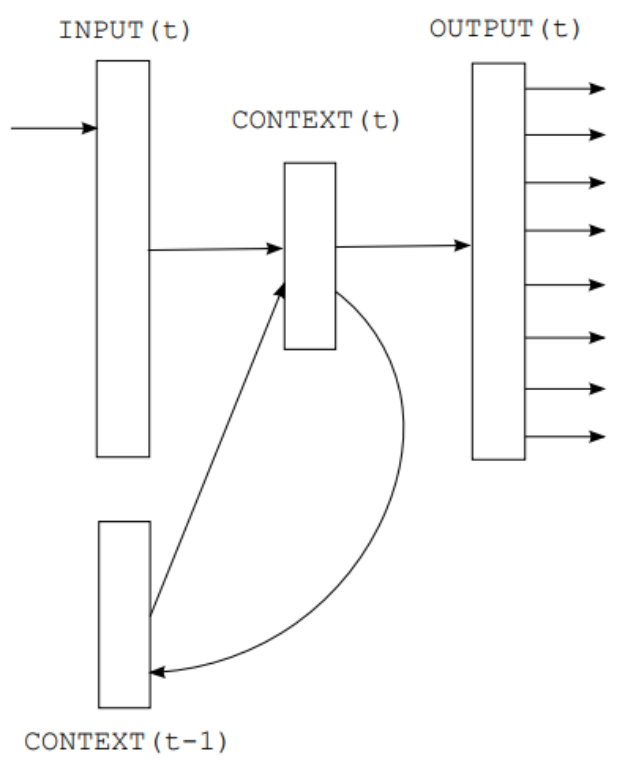

Figure 2.3: A simple recurrent neural networks model, with a single hidden layer (context layer), credit from the authors of [51].

As mentioned previously, RNNs are a specific implementation of these networks. The main difference is present at the input of each layer, where we see the input to a specific node $(x)$ is the concatenation of the current input $x$ at time $t$ with the previous output of that node formed at time $t-1$. Such that we update the neuron formula to match equation 2.3, or as is demonstrated in Figure 2.3 [51].

$$
f\left(\left[x_{t-1}, x_{t}\right] * w+b\right)
$$

For both FNNs and RNNs, weights $(w)$, bias $(b)$ and activation thresholds $(f)$ all need to be set using a supervised learning approach. For RNNs this is achieved using an algorithm known as backpropagation through time. A gradient-based method that unfolds a network backwards across time to calculate the parameters. In certain cases, this lead to the conceptual problem known as vanishing gradient, which occurs when across the chain rule the gradient becomes so small the update to the weights is minimal which can result in the model halting the training procedure [51].

\subsubsection{Long Short Term Memory}

The solution to this problem is achieved by incorporating a Long Short Term Memory (LSTM) approach into the network. The network architecture is modified in such a way that the vanishing gradient problem is avoided, while the actual algorithm is left unchanged. The changes occur within the individual neurons of the model. Within the neuron, three gates are added. Ordered in the following sequence, the gates are titled the forgot gate, storage gate and output gate. The three gates are present within each cell in a model. Where we previously considered an FNNs to be composed of neurons within layers, we now consider the model to be composed of cells which store multiple neuron within layers. 


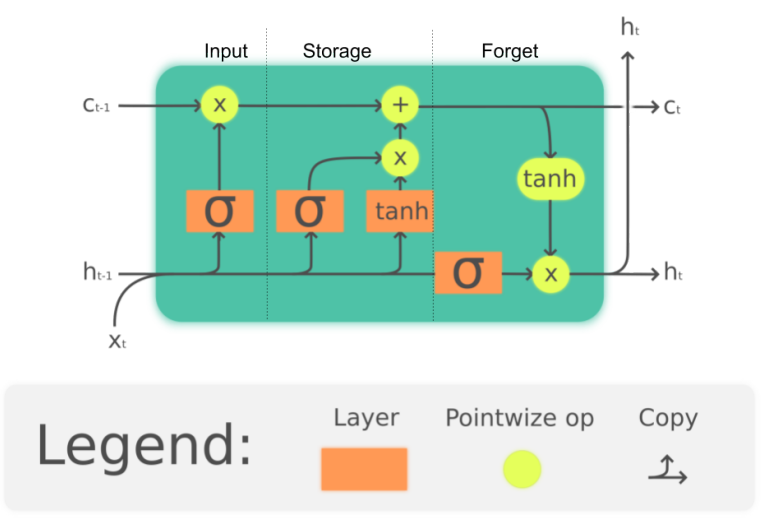

Figure 2.4: A LSTM cell with input, storage and forget gates together.

The forget gate is a normal neuron wrapped in a sigmoid activation layer. For each state in the cell, the output of the neuron is confined to the bounds of 0 to 1 , where 0 indicates that the whole contents of the cell should be forgotten, and 1 that it should be completely kept.

$$
\text { Forget Gate }=\sigma\left(w *\left[x_{t-1}, x_{t}\right]+b\right)
$$

The storage gate, which is affected by the content of the forget gate is next in the sequence. This gate contains two separate neurons, firstly another sigmoid activation neuron is used to determine which content the storage gate well update. The equation matches that of the forget gate. Following this, a tanh layer creates a new vector which will become the new cell state. We then multiple the contents of the input gate by the new cell state to determine which aspects of the cell should be updated. The contents of the forget gate is also multiple by the old cell state to record the new level of information required. We combine these two states to create a new cell state such as demonstrated in equation 2.6.

$$
\begin{gathered}
\text { New empty cell state } / c_{t}=\tanh \left(w *\left[x_{t-1}, x_{t}\right]+b\right) \\
c_{t}=\text { forget gate } * c_{1-t}+\text { input gate } * c_{t}
\end{gathered}
$$

Now that a new cell state has been stored in the cell, the output must be passed to the next cell in the network. The cell is first passed through a sigmoid layer to determine what parts should be passed forward, and then through a tanh layer which bounds all values to -1 to 1 [52].

To further ensure that extremely long sequences contain long term information dependency, two LSTM layers can be used in opposite directions (bidirectional LSTMs) [53]. As demonstrated in Figure 2.5, a bi-RNNs employs at least two hidden layers, each layer feeds information in the opposite direction. The content of the layers are also not shared between each other. As outlined in greater detail in [53], the training method employs both forward and backward passes over the data. This insures that at any given time, both future and past information can be viewed by a neuron to aid in its training. 


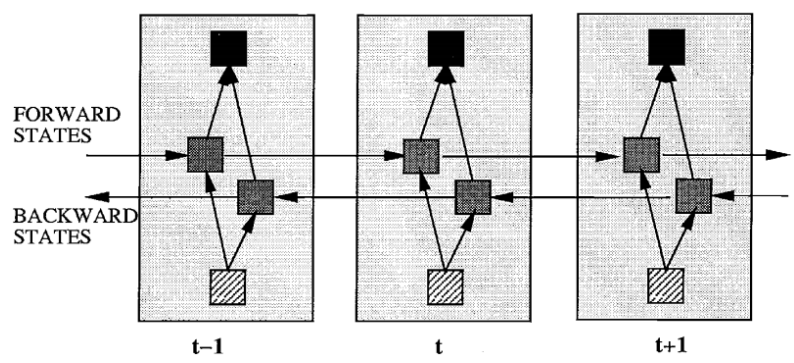

Figure 2.5: A bidirectional RNNs with two hidden layers feeding in opposite directions. Image credit from [54].

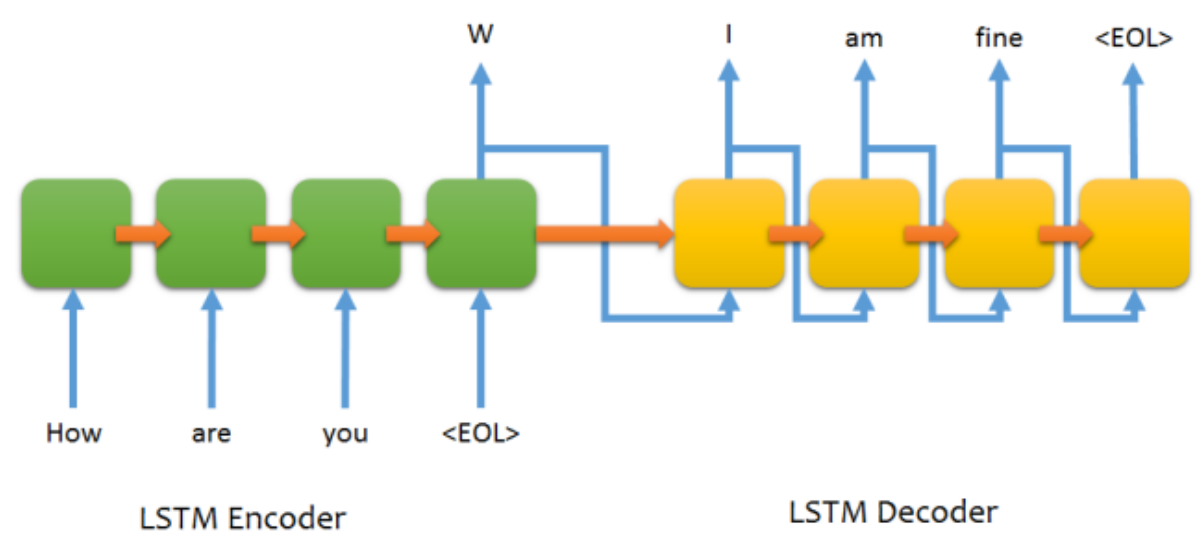

Figure 2.6: A sequence to sequence model composed of four encoders and four decoders. Source; https://github.com/farizrahman4u/seq2seq

\subsubsection{Sequence to Sequence Models}

Given the above possible architecture, sequence to sequence (seq2seq) models are a specific implementation of these networks designed to model one set of data into another. The basis of a seq2seq model is that it contains a set of encoder modules and decoder modules. Each module contains an RNNs style neural network. The number of modules on the encoder and decoder size is fixed. As demonstrated in Figure 2.6, each module on the encoder side accepts two forms of input. An input $x$ from the input content, and a hidden state value $x_{t-1}$. The output of this module is known as a context vector, and become the hidden state value for the next module in the sequence.

For seq2seq models that accept textual data as inputs, the data is first converted to the vector representation of that text. This is achieved by mapping words into a vector space model, which is a high dimensional space where words that share similar semantic meaning are "closer" together. The basis of this is the distributional hypothesis, that words that appear in a similar context share semantic meaning [55]. An embedding space is developed by creating a neural probabilistic language model. By developing a prediction model which employs linguistic and localization parameters to predict word location in multidimensional space [56, 57].

Unlike traditional FNNs, the size of the input vector $x$ can be of arbitrary length. 
In many cases it is set to equal the size of the vocabulary $V$ [51]. However, in larger text corpuses $V$ can be set larger for a more reasonable model to be trained. Therefore, to improve both the generalizability of the model, and the computational training time, data compression algorithms are often employed.

One such approach is byte pair encoding, which begin by dividing the text corpus into character level tokens, while appended each word with a end character " $<\mathrm{w}>$ " to ensure word segmentation is maintained. A number of iterations is chosen, and character are combined to represent the most commonly occurring tokens. For example, if $A$ followed by $B$ is the most commonly occurring pair of tokens, these are replaced by the single token $A B$. The final token vocabulary size is equal to the size of the initial vocabulary, plus the number of merge operation. [54]. This provides better prediction for open vocabularies by reducing the occurrence of rare words.

In addition to an RNNs in an encoder, each module has a self attention layer where information is processed before being passed into the next RNNs. A self-attention layer is a specific vector transformer designed to model dependency of a long vector into a short vector input. For example, if an encoder module accepts a single word as an input, the purpose of the self-attention layer is to model how that word is affected by all the other words in the sentence.

For each word embedding, this is achieved by creating three new values vectors. A query $\left(Q_{x}\right)$, key $\left(K_{x}\right)$ and value $\left(V_{x}\right)$. The content of these three vectors is a linear projection of the embeddings coming into the RNN. A score value is calculated by taking the dot product of $Q_{x}$ and $K_{x}$ and softmaxing the outputted value. The resulting score is then multiplied by the $V_{x}$ vector, and the resulting vector (new word score) are passed to the next RNN [58]. 


\section{3 \\ Related Work}

In this section, we expand the background concepts explained in section two, and move forward by referencing the latest research in each area.

\subsection{Machine Learning Application to Mental Disorders}

The field of health informatics has a long history of developing statistical models to perform prognosis or diagnosis of medical disorders. In general, these models would employ features extracted from biological tests, such as biopsies results being used to predict cancer growth [59]. This approach has seen very little application to mental disorders, due to the fact that many mental disorders have an incomplete aetiology and normally reside at a both a behavioural and more fundamental biological level. Hence, recent research has looked at other areas to detect the manifestation of mental disorders. One such area is psycholinguistics.

\subsubsection{Psycholinguistics}

As far back the 18th century Sigmund Freud proposed that the way in which humans use language reflected aspects of their cognitive processes. Various work over the last thirty years has demonstrated how different cognitive aspects of mental illnesses can manifest in linguistic characteristics. The authors of [60] demonstrated that individuals diagnosed with depression, anxiety and suicidal ideation used a statistically significantly higher percentage of absolutist words. The authors propose this as a manifestation of absolutist thinking, a cognitive component of all three disorders. Similarity, the authors of [61] have shown that those diagnosed with social anxiety disorders have unique linguistical manifestations, notable a higher count of positive words when asked to speak in public.

The largest contribution to this field has been from the author Pennebaker, who has completed a range of studies to demonstrate that individuals diagnosed with depression alter their use of function words compared to non-depressed individuals. This is seen by higher use of singular and personal pronouns, and lower future temporal words $[62,63,64]$. Pennebaker and colleagues propose these are manifestations of Beck's cognitive theory of depression. Concretely, one aspect of Beck's theory is the social integration and disengagement model of suicide, a form of latent thought process. This model proposes that those considering suicide view themselves as 
detached from social life, and not integrated into society. This is manifested linguistically by their higher use of singular and personal pronouns [64].

As an extension of this work, Pennebaker began the process of categorizing various words into psychological constructs. This began the differentiation between content and function words. Function words account for about $0.05 \%$ of the English vocabulary but makeup about $55 \%$ of words used in day to day life. It has been demonstrated that function words reflect how people are communicating, whereas content words convey what they are saying and both types of categories are process differently in the brain [65]. Pennebaker proceeded to categorise various function words into psychological constructs. A total of 104 constructs were formed, with high annotator agreement between all chosen words and categories [66]. This work formed the basis of LIWC, a psycholinguistic dictionary, which assigns certain words to various psychological constructs [66].

Based on the premise of this work, the research community began investigating the possibility of analysing text to perform prognosis or diagnosis of various mental illnesses. Much of this work focused on depression, but also bipolar disorder [67], anorexia [68], and suicide as a standalone disorder [69, 70, 71, 72]. A considerable percentage of the work in this area has originated from the CLEF conference eRisk (Early Risk Prediction on the Internet) workshop, which is in its third consecutive year. The workshop is formed around a shared task, aimed at predicting the presence of certain mental disorders (depression, anorexia, self-harm) based on social media data. Almost 50 proceedings have been published related to this workshop over the last three years. Occasionally other standalone work is published such as that by the authors $[9,73,74]$, but the majority of what we discuss going forward has origination form the eRisk workshop.

\subsubsection{Data}

Data in this area needs to be balanced between two considerations, the size of the data, and the validity of the annotations. Since almost all the publications employ supervised learning, the data requires some form of annotation. The the outcome of these tasks is to predict if an individual is diagnosed with an disorder, the data must be annotated in such a way that it is possible to differentiate between people with the disorder and those without it. One possibility is performing some form of standard clinical diagnosis or screening, often completed through individuals filling out a psychometric screening questionnaire and selecting a cutoff score as indicative of a disorder. This is often time-consuming and can result in smaller sample sizes. Alternatives approaches often employ data mining, either of social media sites [75] or online forums [9]. Generally, the only validation for labelling provided here is making the naive assumption that if an individual talks about certain topics, they are clinically suffering those disorders. For example, much of the eRisk workshop assumes that social media users who post on Reddit forums focused on depression are always depressed, but those who post on forums focused on dogs are not [10]. Although this allows for quantifiability large amount of data to be extract for use in training, the inferences that can be made from this are quite limited.

The majority of these datasets, notable those employed in the eRisk workshop [10] 
and those extracted from twitter [9, 74] consider each individual as belonging to either a binary group, suffering the disorder or not. As the authors of [8] mention, this is a considerable limitation of the resulting work since mental disorders are almost always viewed on a spectrum and binary classification is often considered far to limiting [76].

\subsubsection{Features}

In order to incorporate psycholinguistics into prediction models, the LIWC is almost always employed as a feature extractor, in most cases, all 104 constructs are included $[77,78]$. Occasionally feature selection or reduction will be employed before the training stage which can reduce the number of constructs in certain cases [79]. This collection of psycholinguistic features can be complemented by including additional linguistic measures. Notable features that are repeatedly included are reading scores $[8,78]$, part of speech tagging [80], occasions of drug word usage [81] and sentiment or valence measures [78].

This feature set is then often complemented by a form of text embedding, which is a general attempt at creating a numerical representation of the meaning of words and sentences. This is an active research area within the NLP community, and new state of the art methods are released on an increasingly regular basis. The earliest work in this area made use of unigram or bigram counting approaches [9], which can provide good accuracy despite suffering from the independence problem [82]. To overcome this, more recent approaches often make use of text embedding, which incorporates the distributional hypothesis. The idea that when analysing written text, words that are used and occur in the same contexts tend to have similar semantic meanings [83]. This led to several publication developing their embedding spaces specific to their own datasets, often by employing the Word2vec or Doc2vec algorithms [8, 84], which again improved accuracy scores [78]. The most recent publications have drawn on the latest state of the art methods which incorporate transfer learning. The demonstration that when models are trained on a large corpus and refined on a smaller domain-specific corpus, highest results can be achieved [85]. In this case, commonly used models include ELMO, BERT and the universal sentence encoder $[76,86,87]$.

Many publications will consider the temporal aspect of these disorders in some form, which can either be achieved at an algorithm level or a feature extraction level. Work that makes use of specific data types often employs metadata as features, such as user profile scores, number of previous messages, comment replies etc. At an algorithmic level, time-series approaches have been employed [73].

\subsubsection{Algorithms}

Once a feature space has been created, various algorithmic approaches can be employed. The majority of these approaches employ unsupervised learning [77, 78]. With a limited number of approaches investigating supervised learning such as kMeans Clustering algorithm [88]. Supervised learning often ranges in complexity, from marketability simple models such as Naive Bayes and support vector machines 
(SVM), to deep machine learning approaches. Logistic regression is a popular choice given that the output is easily interpretable [8, 79, 81, 89]. As per the general trend in machine learning, the most recent publications often make use of deep learning architecture $[76,78,80]$. These approaches trade-off interpretability and training time for better accuracy scores.

\subsubsection{Evaluation}

Within the context of the eRisk workshop specifically, the authors [10] propose a specific evaluation measure that balances accuracy of the prediction against the time taken to the make the prediction, rewarding decisions made sooner and penalizing later decisions. This is often reported alongside more traditional evaluations metrics such as precision, recall, accuracy and F1-scores. Certain publications has also computed sensitivity and specificity metrics $[8,76]$.

\subsection{Empathic Module}

As mentioned previously, we can consider empathy as two separate constructs, (i) the ability to detect emotions in others and (ii) the ability to respond to others given knowledge of those emotions. Work in the retrieval field achieves this by hard-coding certain responses that were deemed to be empathic by human experts [11]. However, these systems have no ability to scale and are often time-consuming to develop. The interest, therefore, turns to if empathy could be integrated into neural generation models.

For the purpose of this thesis, we have identified five existing publications in this area to review $[18,34,90,91,92]$. There are three levels at which a neural generation approach can be adapted to incorporate empathy, input, training level or output stages. All these approaches attempt various different alternations to the input stage, with two publications altering the training level and only one the output.

In all cases, the authors attempt to incorporate some degree of emotional understanding within the input stage. This is achieved by one of our two approaches, hard-coded labelling or emotional embedding. The authors of $[18,91]$ use various methods to extract hard-coded labels, often choosing six or eight major emotional classes and annotating each input against a class. The embedding of these classes (often just a one-hot encoded vector) is concatenated against the embedding of the input sentence and fed into a standard training model.

An alternate approach attempts to employ more complex forms of embeddings. The authors of [34] make use of the DeepMoji framework, to creating a $64^{2}$ embedding space composed of a specific emoticon for each label in the space. Again employing a concatenating approach onto the input sentence. Only the authors of [90] attempt to make adjustments to the training stage and output stage to account for the additional emotional vectors. They follow all other authors by concatenating the standard text embedding with a valence arousal dominance model (VAL) composed of the sum of all words in a pre-written VAL dictionary. The standard cross-entropy loss was adjusted to minimize VAL scores in term of Euclidean distance between the response and output. 


\section{4 \\ Methods}

To provide a cohesive overview of the thesis, the section first provides an overview to the development of the psycholinguistic classifier, followed by the development of the empathetic dialogue system and finally the combination of both systems into the experimental protocol.

\subsection{Machine Learning Classifier}

As per Hypothesis 1, this section aims to develop a machine learning classifier that given a text input, or sequence of text inputs can predict PHQ-4 scores. This involves predicting ordinal scores $(0-3)$ for each of the four questions on the form.

\begin{tabular}{|c|cccc|}
\hline \multicolumn{1}{|c|}{ PHQ-4 } & & & \\
\hline $\begin{array}{l}\text { Over the last } 2 \text { weeks, how often have you } \\
\text { been bothered by the following problems? } \\
\text { (Use " } \boldsymbol{N} \text { " to indicate your answer) }\end{array}$ & $\begin{array}{c}\text { Not } \\
\text { at all }\end{array}$ & $\begin{array}{c}\text { Several } \\
\text { days }\end{array}$ & $\begin{array}{c}\text { More than } \\
\text { half the } \\
\text { days }\end{array}$ & $\begin{array}{c}\text { Nearly } \\
\text { every day }\end{array}$ \\
\hline 1. Feeling nervous, anxious or on edge & 0 & 1 & 2 & 3 \\
\hline 2. Not being able to stop or control worrying & 0 & 1 & 2 & 3 \\
\hline 3. Little interest or pleasure in doing things & 0 & 1 & 2 & 3 \\
\hline 4. Feeling down, depressed, or hopeless & 0 & 1 & 2 & 3 \\
\hline
\end{tabular}

Figure 4.1: Patient Health Questionnaire (PHQ-4), four item version.

\subsubsection{Data}

As mentioned in Section 3, there is an ongoing challenge between finding sufficiently sized and appropriately annotated datasets. For the purpose of this thesis, we were provided with permission to use the DAIC-WOZ dataset [47]. The dataset is composed of transcribed clinical interviews collected through a Wizard-of-Oz approach ${ }^{1}$

\footnotetext{
${ }^{1}$ Human talking to a computer program which is controlled in realtime by another human being
} 


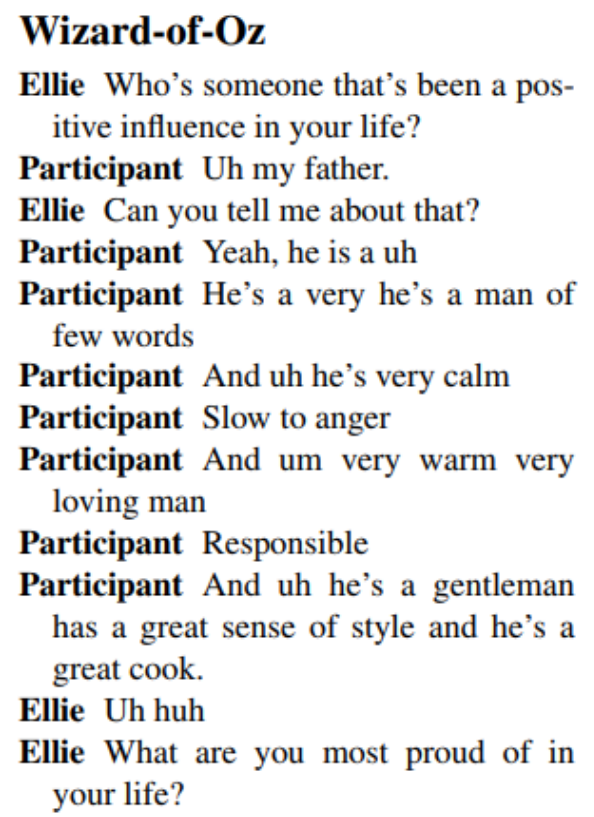

Figure 4.2: An extract of the DAIC-WOZ dataset, image credit from [47]

for 142 patients. For all interviews, the topics of the conversations were general nonspecific topics and all were collected within the United States.

In addition to the transcripts from each patient, PHQ-8 questionnaires were administered at the same time as the interviews and these results were provided. For the purpose of our task, we combined all statements across all patients into a single dataset, furthermore, all non-patient statements (from the Wizard-of-Oz system) were removed leaving a total of 23,726 text statements.

This dataset is the largest publicly available dataset that contains PHQ scores matched against text transcripts, based on the literature review in Section 3, we feel it is best to place emphasize on valid annotation compared to possible increased data sizes that could be found in social media data.

As hypothesis one states, the outcome of our prediction system is PHQ-4 scores. The PHQ-8 and PHQ-4 share two questions, thus we can directly select these from the data provided in the DAIC-WOZ dataset. However, two PHQ-4 scores are missing, these two questions and corresponding scores concern anxiety, and are commonly refereed to as the GAD-2 questions ${ }^{2}$

As outlined in Section 2.1, both anxiety and depression share the same fundamental aetiology only differentiating in the trigger activation of certain symptoms. With such a strongly defined comorbidity and underlying shared aetiology, we proposed investigating if GAD-2 scores could be predicted from answers to the PHQ- 8 scale.

\subsubsection{Predicting GAD-2 from the PHQ-8}

To achieve this, we were provided with a dataset from [93] containing 1,307 PHQ-8 and GAD-2 scores from a national Swedish sample. The distribution of the scores within this sample matched previous population samples.

\footnotetext{
${ }^{2}$ GAD abbreviation stands for generalized anxiety disorder.
} 
Table 4.1: Distribution of values for GAD 1 and GAD 2 questions within the dataset

\begin{tabular}{l|llll}
\hline \hline & 0 & 1 & 2 & 3 \\
\hline GAD 1 & 657 & 497 & 81 & 72 \\
\hline GAD 2 & 1,042 & 196 & 38 & 31 \\
\hline \hline
\end{tabular}

We approach this task as a classic prediction problem, given a set of PHQ-8 scores, predict GAD 1 and GAD 2 scores. PHQ and GAD are ordinally encoded, and therefore it makes sense to consider the values 0,1,2 and 3 as independent classes. Our first approach focused on multi-class algorithms, however as per Table 4.1 shows, there is an uneven class balance among both datasets. A range of approaches have been proposed within the literature to deal with uneven class balance specifically within psychometric datasets [94, 95]. For the purpose of this project, we decided to approach the problem as a three-step pipeline.

During exploratory data analysis, we observed that in $87 \%$ and $92 \%$ of cases, if all PHQ-4 values were equal to zero, both GAD scores were equal to zero as well. We considered this to be a sufficiently high accuracy score that we could re-code all GAD 1 and 2 scores as zero if the above case was present. This was the first step in our classification pipeline.

To balance the uneven class problem, we recorded the four classes as one of two classes. Either 0 or $(1,2,3)$. This provides a more even distribution closer to a $50 / 50$ split, (step two). In the situations were our classifier predicts the $(1,2,3)$ score, an optional third step was applied to predict which of these classes (1 or 2, or 3 ) are appropriate. In total four classification models were required to be trained to complete these three steps across two questions.

Listing 4.1: Pseudo-code for prediction pipeline

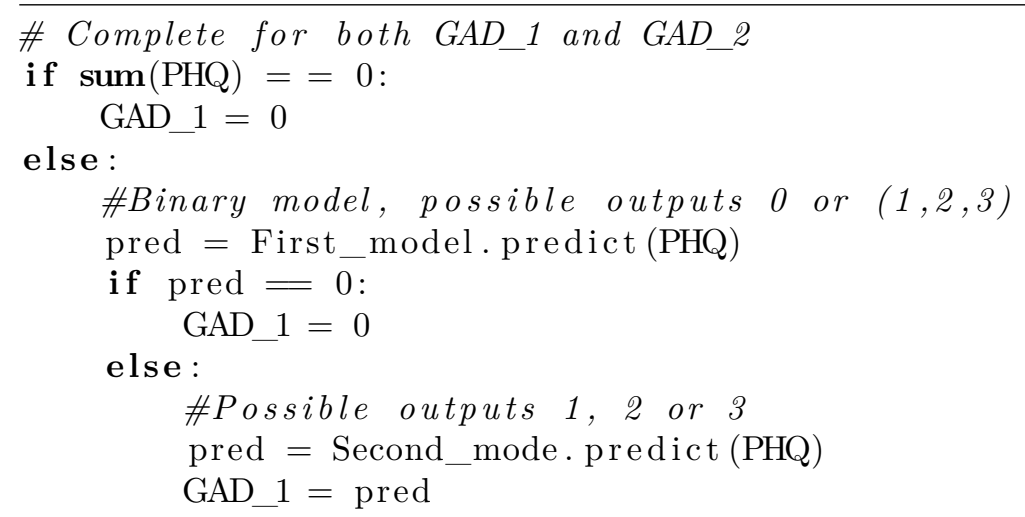

To match the above pipeline, we removed all cases were PHQ scores and GAD scores equal zero from the dataset. The resulting dataset was then recoded with all cases of 1,2 and 3 being relabeled as a single value. A second dataset was created of just those observations which had a score of 1, 2 or 3. Both datasets were divided into $80 \%$ cut for training, $10 \%$ for testing and a final $10 \%$ for evaluation.

For both datasets, we chose to employ a multi-layer perceptron classifier, hyperparameters were set using a grid search technique on the first of our classification problems. The following hyperparameters were set, alpha $=0.00001$, number of hidden layers $=11$, activation function $=$ relu, learning rate $=$ constant. For each 
Table 4.2: Features for each GAD classification problem, features are numbered PHQ-8 questions

\begin{tabular}{l|l}
\hline \hline Classifier & Features \\
\hline GAD 1 / 0 or $(1,2,3)$ & $2,3,5,6$ \\
\hline GAD 1 / 1,2 or 3 & $4,7,8$ \\
\hline GAD 2 / 0 or $(1,2,3)$ & $2,3,4,6,7$ \\
\hline GAD 2 / 1,2 or 3 & 1,7 \\
\hline \hline
\end{tabular}

Table 4.3: Accuracy results of each of the four classifiers.

\begin{tabular}{l|lllll}
\hline \hline & \\
\hline Majority class baseline & 0.63 & 0.77 & 0.73 & 0.66 \\
\hline Overall accuracy score & 0.71 & 0.80 & 0.84 & 0.70 \\
\hline Class 1 accuracy score & 0.70 & 0.95 & 0.93 & 0.98 \\
\hline Class 2 accuracy score & 0.72 & 0.19 & 0.62 & 0.12 \\
\hline Class 3 accuracy score & & 0.38 & & 0.15 \\
\hline
\end{tabular}

classification problem, we optimized the feature selection by performing an exhaustive search for all combinations of eight input features and selecting the combination that returned the highest accuracy score.

The following combination of features was selected for each of the four classifiers (Table 4.2). Results of the four classifiers on the evaluation set are presented in Table 4.3 .

This three step approach described above was then applied to the DAIC-WOZ dataset to predict GAD-2 scores for each participant in the study.

\subsubsection{Feature development}

Given the DAIC-WOZ text data, and matching PHQ-4 scores. The following subsections describes the feature processing completed on the data. We employed three distinct methods to create our feature space.

\subsubsection{Text Embedding}

Text embedding was employed using a pre-trained implementation of the universal sentence encoder (USE). The USE approach is designed for longer strings compared to other pretrained models which work on a single word or token level. A transformer architecture is applied to each string to model dependence across the length of the string and compute a context aware word representations. The authors of the paper release a pre-trained implementation trained on a large body of generic data strings 
$[96]^{3}$.

We chose to make use of USE for two reasons, firstly that the format of the DAICWOZ is mostly in single sentences. Our desire was to capture the whole semantic meaning of the utterances, and sentence level representing was felt preferable to combining single word level. Secondly, given the small nature of the DAIC-WOZ, using a pre-trained model allowed for better generalisability of the model to unseen new data. Each of the patient statements was passed into the pre-trained model and a statement-level representation vector of shape 512 was returned.

\subsubsection{Psycholinguistics}

To model psycholinguistic features we employed the LIWC library, which is a psycholinguistic dictionary containing 94 psychological trait dimensions and over 2,000 words related to these dimensions [63]. A percentage count of the number of words in the text related to each dimension is computed. To identify an optimal subset of the number of relevant dimensions, we reviewed all proceedings from the CLEF eRisk workshop 2017 and 2018. For each proceeding that employed LIWC, the list of dimensions included was taken. An intersection of these lists was then taken to create a subset of 22 relevant dimensions, which resulted in the following features being included in our model: word count, analytical thinking, authentic, emotional tone, function words, pronoun, personal pronouns, 1st person singular, 1st person plural, 2nd person, 3rd person singular, articles, auxiliary verbs, conjunctions, negations, regular verbs, negative emotions, social words, cognitive processes, past focus, present focus, future focus.

\subsubsection{Psychometric similarity}

Recent work has seen success in comparing word embeddings in terms of semantic similarity $[97,98]$, were the distance between embeddings in a $x^{N}$ dimensional space is considered equal to their likeness in terms of the semantic content. Since USE creates sentence level embeddings and is trained on a large cross-domain corpus, it allows us the ability to compare sentences in terms of similarity. We employed this approach by comparing the semantic similarity of patient statements with responses from psychometric questionnaires. The principle was that if a patient statement reflected the same content of a psychometric test it should have a higher similarity score compared with a random statement.

Four questionnaires were identified by choosing cognitive theories relevant to the aetiology of each of the four PHQ-4 symptoms. Explanation of this is provided in Section 2 of this thesis, and in Table 4.4. The concatenation of questions across all four questionnaires amounted to 102 questions. For each patient statement, a 512 embedding dimension was computed with the USE pre-trained model, along with this, embeddings for each of the 102 patient questions were computed.

Distance between vectors in the embedding space was measured using cosine similarity (CS), where two vectors are compared from a single starting point in space in terms of angle. A CS of 0 indicates orthogonality of the the two vectors, and

\footnotetext{
${ }^{3}$ The model was extracted from Tensorflow hub on the 10/04/2019 https://tfhub.dev/google/universal-sentence-encoder/2.
} 


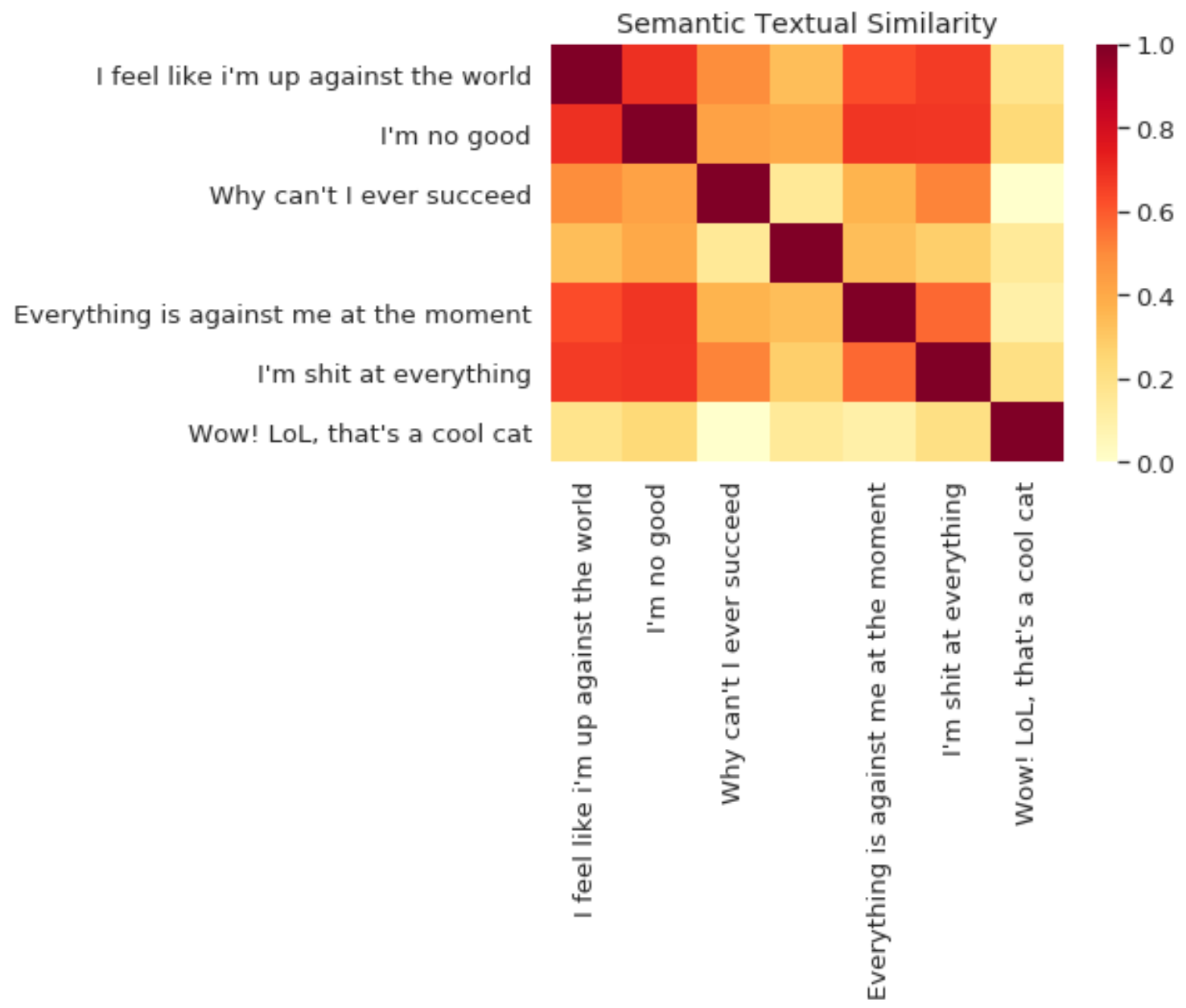

Figure 4.3: Demonstration of semantic similarity using a heat map. Sentence 1,2 and 3 are extracted from psychometric questionnaires. Sentence 4,5 are highly similar to these questions, while 6 is not. 
Table 4.4: Summary of aetiology theories and assessment tools

\begin{tabular}{lll}
\hline \hline PHQ-4 Symptom & Theory & Assessment tool \\
\hline Feeling nervous or anxious & Intolerance of uncertainty [23] & $\begin{array}{l}\text { Intolerance of } \\
\text { Uncertainty Scale }\end{array}$ \\
\hline Uncontrollable worry & Positive belief about worry [23] & $\begin{array}{l}\text { Penn State } \\
\text { Worry Questionnaire }\end{array}$ \\
\hline Anhedonia & Avoidance behaviour [23] & $\begin{array}{l}\text { Cognitive-Behavioural } \\
\text { Avoidance Scale }\end{array}$ \\
\hline Depressed mood & Negative triad [21] & $\begin{array}{l}\text { Beck's depression } \\
\text { inventory }\end{array}$ \\
\hline \hline
\end{tabular}

therefore little relation between them. Figure 4.3 shows a heat map demonstration of CS scores across related and unrelated vectors.

\subsubsection{Feature space}

The resulting dataset was composed of 636 features. All features were scaled by removing the mean and scaling to unit variance within the bounds of -1 and 1 .

\subsubsection{Algorithm identification}

As mentioned previously, the desired output of the prediction system is to be able to predict an ordinal value for each of the four separate symptoms on the PHQ- 4 . Previous work has demonstrated that a single deep neural network (DNN) can model the interconnectivity of a problem space better than separate individual classifiers [99].

For each patient statement, the neural network needs to be able to output an ordinal value score for each question. This requires that the network outputs both multilabel (four symptoms) and multilabel (ordinal score). This architecture is regarded as multi-dimensional or multi-targeted classification, were the output is assigned both a set of labels $y=\left(y_{0}, \ldots, y_{d}\right)$, and for each label $y$ an ordinal value in the range of 0 to $d[100]$.

For the purpose of this work, we proposed a two-step approach. We begin by training a multilabel algorithm, the specific architecture of which is described in the following subsection. However, in order to ensure the model appropriately deals with multi-label classes, a sigmoid cross-entropy loss function is applied ${ }^{4}$. This is a modification of a normal cross-entropy loss function, as shown as in equation 4.2, were values are first passed through a sigmoid function (as per equation 4.1)

$$
f\left(s_{i}\right)=\frac{1}{1+e^{-s_{i}}}
$$

$$
\text { Cross-entropy }=-\sum_{i=1}^{C^{\prime}=2} t_{i} \log \left(f\left(s_{i}\right)\right)=-t_{1} \log \left(f\left(s_{1}\right)\right)-\left(1-t_{1}\right) \log \left(1-f\left(s_{1}\right)\right)
$$

\footnotetext{
${ }^{4}$ Also known as binary cross-entropy loss.
} 


$$
\begin{aligned}
\text { where } t & =\text { Ground truth } \\
s & =\text { Predicted score }
\end{aligned}
$$

The above set up is computed independently for each class $c_{i}$, furthermore, the problem is divided into $x$ binary comparisons between all classes. The loss is summed up over the different binary problems, while the gradients are summed to backpropagate the whole process $[80,101,102,103]$.

The final output of the above algorithm is a sigmoid score for each of the four possible symptoms. We manually assign three threshold levels, to classify the output score in terms of ordinal values. The following threshold levels were employed; if the outcome of the sigmoid function is less than 0.25 , an ordinal score of 1 is applied, if the Sigmoid score is between 0.25 and 0.50, 2 is applied, between 0.50 and 0.753 is applied, if the Sigmoid score is larger than 0.75 a score of 4 is applied.

To compare our approach against a simpler model architecture, and determine if a DNN architecture is appropriate, we also trained a random forest classifier which is equally able to model multi-label outputs [104].

\subsubsection{Evolutionary tuning}

Tuning possible hyperparameters (HPs) is a vital aspect for achieving a model that correctly fits the problem space. Given training time, and the number of possible unique HP combinations, its computationally inefficient to perform an exhaustive search [105]. Evolutionary tuning is a subfield of genetic programming. It provides a more optimized approach to finding a suitable set of HPs by employing concepts from evolutionary biology [106].

During the biological evolution of a complex organism, when an offspring is born, there is the possibility that the specific combination of genes that occurs can combine in a previously unseen way or unexpected way, this is known as a mutation. These can either be advantageous or not, depending on if the mutation increase the chance of survival for the being. Within computer science terms, a mutation is judged on its effectiveness by use of a chosen metric (known as a fitness function).

To apply this process to our problem, we began by choosing a broad set of possible HPs (details in appendix 2). A set of twenty neural networks were trained, each composed of a random subset of HPs. Each network is evaluated based on our fitness function, the minimization of the Hamming loss criteria [102] ${ }^{5}$. The five best performing networks are chosen, along with three random others from the remaining 15. This is to ensure a certain level of variability in the population in hopes of an advantageous mutation.

The fundamental architecture of the model we employ is a multilayer perceptron algorithm, composed of a minimum of one input layer (neurons $=$ size of feature vector), one hidden layer, and a final output layer (neurons $=4$ ). In all cases, the models were trained for 50 epochs. A dropout of 0.2 was applied to all hidden layers that were added. The following hyperparameter categories were then optimized:

\footnotetext{
${ }^{5}$ The Hamming loss is the fraction of labels that are incorrectly predicted. Given four label, an HL of $.25 \%$ suggest one label is incorrect.
} 


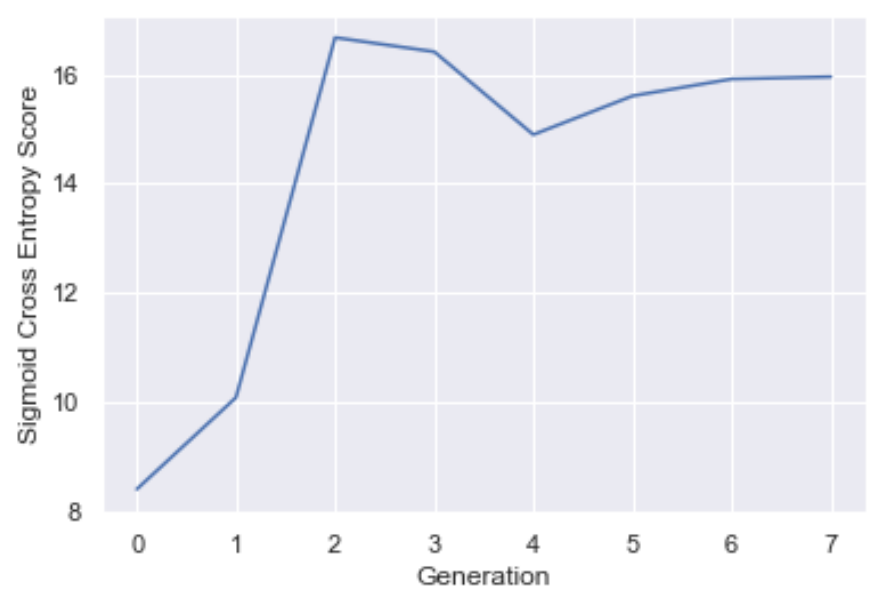

Figure 4.4: Average sigmoid cross entropy score for each generation across the training

the number of hidden layers, the number of neurons, the activation and optimizer functions.

We train eight generations of networks, generations are trained in a sequential order, and within each generation twenty different neural networks are trained each composed of different HPs. For the next generation in the sequence, we carry over seven existing networks as explained above. To create an additional 13 we select random combinations of hyperparameters from the seven first networks and combine these in 13 unique ways. Our aim is to find a mutation that is advantageous. All networks are trained and evaluated again, and the process repeated. A graph of sigmoid cross entropy value across generations in presented in Figure 4.4

The final optimal HPs, based on the minimized Hamming loss, were five hidden dense layers with dimensions of 1024, 768, 256, 128, 64 in that order. Each layer contained a relu activation function, except for the final layer, which contained Sigmoid. Binary cross-entropy was applied to compute the loss function and adagrad function as the optimizer.

The following HPs were employed for the random forest classifier (RFC), number of trees in the forest $=10$, split criterion $=$ gini, no max depth of trees, minimum samples to split a tree $=2$, minimum leaf sample $=1$.

\subsection{Dialogue System}

The following section provides an overview to our approach for Hypothesis 2. As explored in Section 2.3, empathy can be broadly viewed "as the ability to recognize emotions in others and react appropriately". Representing emotions in language has long been a topic of interest to NLP researchers. As reviewed in Section 3.2, previous approaches include categorical labelling of emotions (Happy, Sad, Fear), embedding space vectorization of emotional labels, or sentiment embeddings. One limitation seen with the previous attempts to combine emotions with dialogue to 


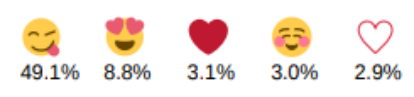

Figure 4.5: Sample annotion from the DeepMoji framework, showing the five highest probability emojis for a specific text input.

model empathy is the limited use of emotions in annotating the dialogue utterances. In most cases, a single one of Elkman major six emotions is used [91]. Firstly, this assumes that all human connections can be modelled in $x$ number of emotions which has been shown to be false [107]. Secondly, this implies that each dialogue utterance can only represent a single emotion.

DeepMoji is an annotation framework that has seen growth in popularity over the last year [108]. The authors of this work use a novel method to extract a text corpus from twitter that contains emojis ${ }^{6}$. Using a DNN approach, they developed a model that predicts (or annotate) text inputs with the probability that the input represents each of the 64 most popular emojis used on English speaking twitter. A sample annotation is provided in Figure 4.5 displaying the emojis with the five highest probability for a specific text input.

Within the context of our work, DeepMoji offers an interesting perspective on emotional labelling. Emojis are not direct labelling of human emotions [108], their use is considered to be more fluid and may adapt over time. Instead of a top-down labelling of the observed behaviour, bottom-up approaches were developed to match certain behavioural patterns [109]. As a result, the authors of [108] argue that by leaving them undefined, they provide a more realistic annotation of human feelings and emotions. However, certain work which has attempted to label them within Elkman emotions [109].

As per the definition mentioned above, we propose that "the ability to recognize emotions" will be handled by the DeepMoji framework. This means that for each user input, the framework will annotate the input with its predicted emojis (three most likely). To model the "react appropriately" aspect, we propose using a seq2seq data-driven approach. As discussed in Section 2.5.1, a standard seq2seq model can be used to generate dialogue utterances in a conversation. The question therefore concerns how to train this approach to "react appropriately" to emotions.

We propose achieving this by adapting the approach proposed by the authors in [110], originally designed to train multilingual, multiway neural machine translation models. When developing a model to translate a single language, the data and architecture is normally such that a source language is passed into an encoder model, and a target language is passed into a decoder model. Backpropagation through time is used to train both the encoder and decoder to appropriately predict this language. The multiway model is proposed to overcome the limitation of dealing with a smaller dataset that are common for low resourced languages. The approach begins by using a standard parallel aligned translation corpus with multiple languages within the same corpus. Each parallel alignment is predated with a source and target language

\footnotetext{
${ }^{6}$ Emojis which are a picture or an icon-based version of the text emoticons. They are standardised by the Unicode consortium internationally. Examples can be seen in appendix 1.
} 
token as per Equation 4.3.

$$
\underbrace{E N->F R}_{\text {Source and target language tokens }} \text { Hello world } \rightarrow \text { Bonjour Monde }
$$

Where the left side of the arrow is the source dataset and the right side is the target dataset. (4.3)

Compared to a single language translation model, with one encoder and one decoder architecture, the multiway model has $N$ encoders, $M$ decoders and a shared attention mechanism. Both $M$ and $N$ are set by the number of source and target languages. For example, if the parallel corpus has four languages on the source side, four separate encoders are trained. Meaning that a single translation model is used for translating the source language to the multiple target languages.

$M$ and $N$ are set by the number of source and target languages. One encoder and one decoder exist for each source and target language. Meaning that a single encoder or decoder is shared for translating the language to multiple target languages. However, a single attention mechanism is employed across all encoder and decoder language pairs. The experiments completed by [110] demonstrate that this approach performs well for low resource language, since the approach captures both language-specific aspect and combines general language characteristics.

This architecture allows a general seq2seq model to specifically adapt to a given context. In the same way, a multiway seq2seq model would use the format shown in Equation 4.3, our proposal is that by replacing the source and target language tokens with source and target emoji annotations, we can direct the network to incorporate emotional context in the dialogue generation.

Practically this results in training $N$ number of encoders, one for each emotional context in the source dataset and $M$ decoders for each emotional context in the target dataset. While the shared attention layer is general for the whole dialogue dataset. To prevent a unreasonable number of encoder and decoders being trained, we propose methods to reduce the emotional contexts in Section 4.2.1.2.

We observed that the approach satisfies both the conditions of "the ability to recognize emotions in others" and "react appropriately". A visual representation of the data format is provided in Figure 4.6.

\subsubsection{Data}

\subsubsection{OpenSubtitles}

The OpenSubtitles dataset is an online collection of subtitles for major movies worldwide $[111,112]$. The data is provided in an XML format, ordered by year and encoded with a timestamp and the tokenized subtitles text. We created an aligned dataset by assuming if a subtitle was spoken within one second of the previous subtitle they could be aligned for dialogue consistency, this approach has been seen before from the authors of [8]. We extracted all English subtitles from the corpus and in total generated 80 million parallel dialogue utterances. For the purpose of this work, we selected the first 10 million of these to continue to work with. OpenSubtitles 

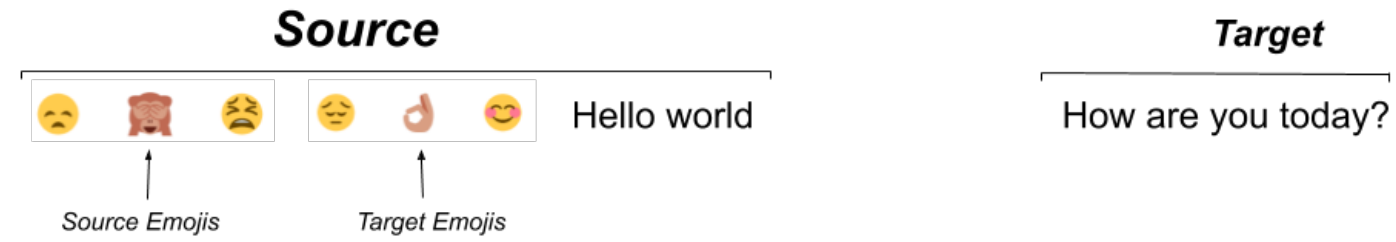

Figure 4.6: Visual representation of our empathic training approach. Emojis are represented by a single token of type integer_integer_integer

is the largest human-to-human non-domain specific freely available dataset. The advantage of the dataset is the size of the data and the wide domain range of the topics and vocabulary.

\subsubsection{DeepMoji}

For the purpose of this project, we made use of the pre-trained DeepMoji model released publicly by the authors of [108]. We passed the 10 million OpenSubtitle source and target sentences through the annotation model and returned the three most likely emojis as each input. Emojis are represented as a single integer of 0 to 63, for more details see appendix Figure A.1. These three emojis were concatenated to form a single token such that 636363 would become "63_63_63".

Concatenating reduced the variability of the number of possible tokens and data sparsity. This was hoped to improve training by strengthening certain token utterances. Given more training data, we wouldn't have aimed to reduced data sparsity in this manner. Text input format was UTF-16 encoded.

To further reduce variability in the model, we apply a rare word encoding approach. We combine both source and target annotations and plot the distribution of all tokens (Figure 4.7). We selected the first 30,000 most occurring tokens, and relabeled all other tokens as an artificial rare word token "64_64_64". The number of relabeled tokens is less than the accumulative 30,000 tokens.

These tokens were appended to the training data after BPE had been applied to the text data.

\subsubsection{Dialogue Language Model}

To train our generative language model, we employed the OpenNMT framework [113]. Our training dataset was ten million dialogue pairs in the format shown as Figure 4.6. A byte pair encoding algorithm was applied to both the source and target textual data [54]. The resulting sentence source data was then predated with 


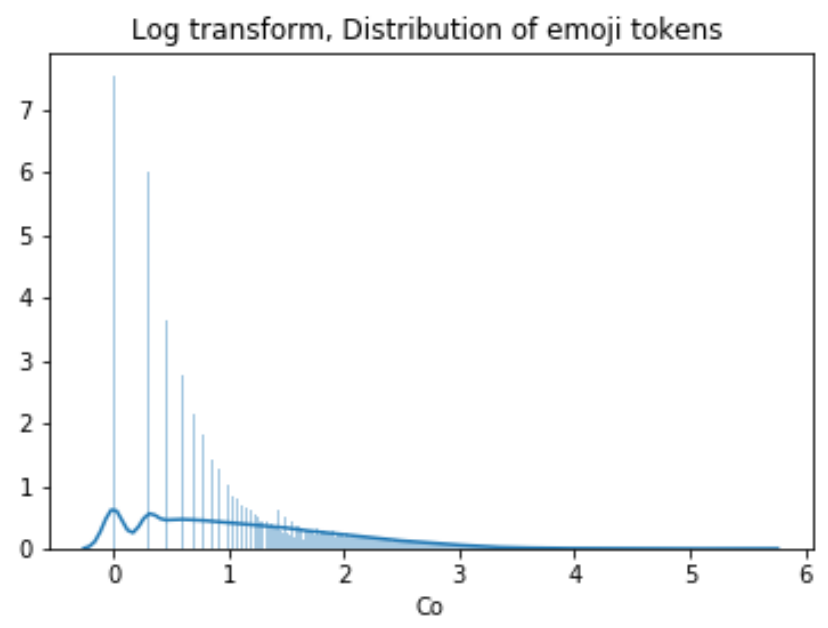

Figure 4.7: Distribution of emoji tokens across both datasets

source and target emojis.

We applied a seq2seq model, with a bi-directional LSTM training algorithm of two hidden layers, 500 hidden bidirectional LSTM units, input feeding enabled, batch size of 64, 0.3 dropout probability and a dynamic learning rate decay. Our dataset was divided into an $80 \%$ training cut, $10 \%$ testing and $10 \%$ evaluation. The model was trained for 13 epochs, whereby the perplexity scores for each of the epochs are presented in Figure 4.8.

For the purpose of evaluation, a non-empathic dialogue system was designed using the same architecture and dataset, with the exception of the emoji tokens.

\subsubsection{Implementation Pipeline}

For the purpose of the public evaluation, an implementation pipeline was required which would allow the dialogue language model to be run in real-time. The pipeline begins with a user inputting text, which is annotated for emojis using the DeepMoji system ${ }^{7}$ This provides us with the source text and source emojis. For the multiway model, we additionally require the target emojis to inform the system of how to direct the dialogue generation.

To achieve this, we trained a naive prediction system. For each source token in the annotated OpenSubtiles dataset (from Section 4.2.1.1) we select the highest probability target token shown in the dataset, such as per Equation 4.4. This provides us with a reference table of 30,000 source token and corresponding most probable target tokens. Unseen source tokens are assigned a unique value token of "64 64 64".

$$
\text { Source }=\max (P(\text { Target }))
$$

Within the pipeline, a source token is looked up within the reference table and the most probable target token is selected. The source and target token, along with

\footnotetext{
${ }^{7}$ For the purpose of the project, the released model was merged with a Keras Rest API to allow inferences in real-time.
} 


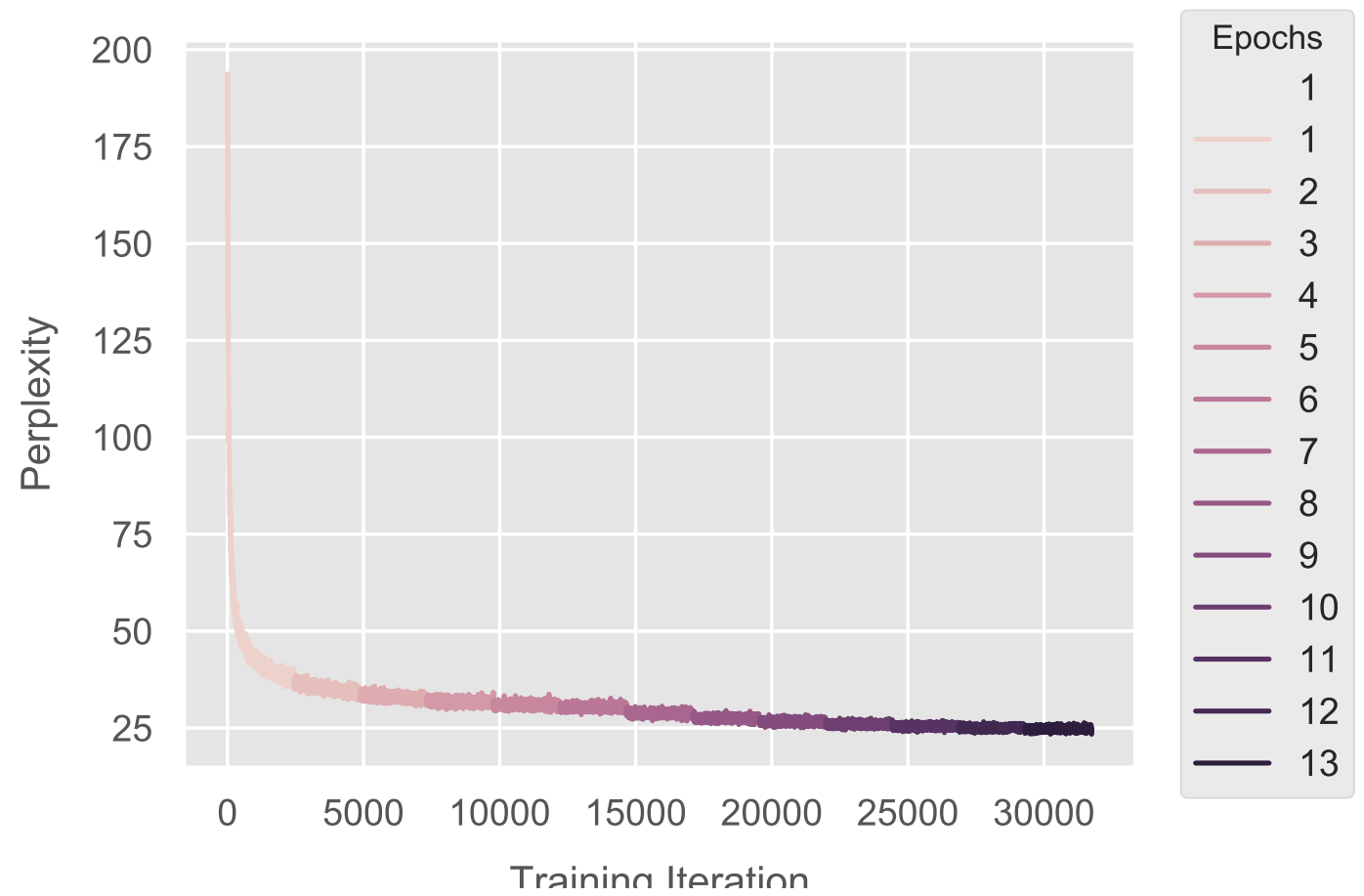

Figure 4.8: Perplexity score across training 13 epoch iteration

the source text are then all concatenated and interfered into the dialogue language model. This output is the target text and passed to the user.

To ensure dialogue consistently across the user conversation, each input to the model is a concatenation of the last two inputs plus the current one. Where input is the "Source Emoji token + Target Emoji token + Source text". This follows the work of the authors [8].

\subsection{Evaluation system}

To evaluate both hypotheses, a public-facing experimental system was required. Given the nature of the project, and current concerns regarding data protection, there was a requirement that the system was designed in house as opposed to using an external platform. The system was completely developed by the student of this thesis. For security reasons, it was launched on an institutional virtual machine server. The front end of the program was written in PHP and JavaScript, with a backend system written in python and PERL.

Participants were recruited by convenient sampling. Invitation to participate were distributed across institutional and international mailing lists. Participants were offered the possibility of entering a raffle for a prize in return for completing the study. The system maintained the anonymity of participants as per rectal 32 of the GDPR framework. Participants could complete the study from any location provided they had constant internet access. Once landing on the web page, they were provided with the study information sheet detailing aspects such as the pur- 
Table 4.5: Questions to evaluate the empathetic module. All questions were answered on the same three level Likert scale (Not at all, Somewhat so, Very much so)

1. Did the responses from the system show understanding of your feeling / experiences?

2. Where the responses seem appropriate to the conversation? (Were they on topic)

3. Could you understand the responses? (Did the language seem accurate)

4. Did you feel trust toward the system?

5. Did you feel the system understood your emotions?

6. Did you feel the system had emotions?

pose of the experiment, requirements and rights of the participants and ethical and legal information. If participants choose to continue, they were provided with an eight form questionnaire to access eligibility. Inclusion criteria required that the participant were over the age of 18 years and could speak a working level of English. Failure to meet these criteria resulted in exclusion.

If a participant did not consent to anonymous data collection, they were offered the ability to talk to the dialogue system but no data was collected. If a participant met all criteria, they were randomly allocated to one of two trained dialogue systems. They were asked to have a short conversation (at least 20 messages) with these systems on any topic they wished. There was no counter system in place, so it was the requirement of the individual themselves to monitor this. A profanity filter was installed on the system to replace words deemed as inappropriate with asterisks.

On completion of the conversation, participants completed the PHQ-4, and four questions concerning the empathic aspect of the system (shown in Table 4.5). Participants were free to leave the study at any time, and were offered a participant distress protocol page if they did so. A full diagram of the experimental protocol is displayed in Figure 4.9. The system employed anonymous ID codes to ensure that all participants followed the same route, if a participate left and returned to the study it was considered a new participate. Screenshot of all pages displayed to the participants are presented in appendix 6 .

\subsection{Data Protection}

Given the nature of the thesis, ethics and data protection were of central consideration at all stages. As the thesis work was completed while part of the Insight Centre for Data Analytics, whose parent institute is NUI Galway. The project was required to adhere to all ethical and data protection requirements of the university. According to Section 3(2) of the Irish Data Protection Act 2018 (Section 36(2) Health Research) Regulations $2018^{8}$. , the work undertaken within this thesis is considered to be health research and therefore must adhere to the requirements of

\footnotetext{
8 This statue is an regional implementation of the European general data protection regulation $2016 / 679$
} 


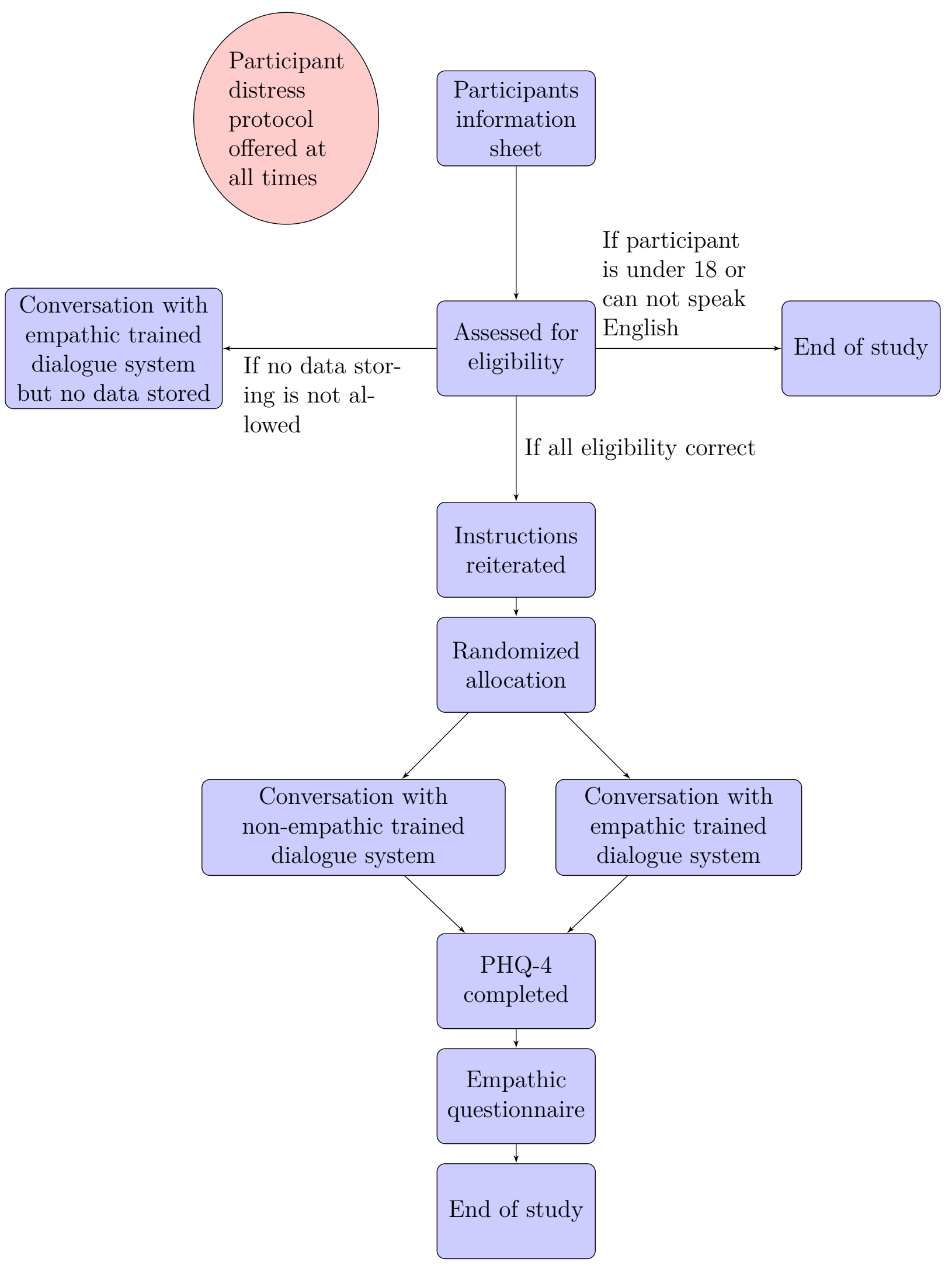

Figure 4.9: Diagram of experimental protocol employed. Followed from a top down approach. 
this statue [114].

The statue requires two formal submissions to be made, firstly a Data Protection Impact Assessment (DPIA) to both the research centre and university data protection officers and an ethics application to the university research ethics committee (REC). The DPIA is a formal submission concerning the collecting, storage and processing of data that take places within this project. The legal basis for processing data as part of this project were GDPR article 6 subsection $1(\mathrm{~A})$ and article 9 subjection 2(i). The DPIA was approved by both officers by March 2019 following recommended feedback changes.

The ethics application is with specific regards to the human evaluation aspect of the thesis, the application concerns the process of recruitment, what participants will undergo as part of the experiments and the possible risks involved, as well as long term care and support. All participant recruitment material, information sheet, distress protocol and questionnaires were submitted for review. The ethics board approved the study on the 21/04/2019 and written confirmation can be seen in the appendix within Figure C.1. Participant information sheet can be found in appendix 5 and participant distress protocol in appendix 6 . 


\section{5 \\ Results}

\subsection{Multi-label Classifier Training}

The following are results from the training of the machine learning classifier to predict PHQ-4 scores as described in Section 4.1. For this subsection, all evaluation comparison is on the DAIC-WOZ dataset. As per Section 4.1, the results presented here are calculated on a $20 \%$ held out evaluation dataset, which was distinct from the training and testing datasets.

\subsubsection{Multi-label}

Our first evaluation was performed on the multi-label aspect of the network. DAICWOZ PHQ-4 scores were reduced to a binary class (0 for 0,1 for $1,2,3$ ) and Sigmoid outputs were binarized on a cutoff point of 0.5. Hamming loss was the chosen metric for evaluation, which computes the distance between predicted and true values. A ten-fold cross-validation resulted in a score of 0.388 , with a $95 \%$ confidence interval of $[0.3870,0.3905]$. To compare this against a random baseline, where a set of prediction scores are computed using a random number generator, a Hamming loss of $0.49,95 \%$ [0.481, 0.519], is achieved. Hamming loss is an inverse metric, and minimization of the score is desired.

\subsubsection{Multidimensional}

Using the cutoffs mentioned above in Section 4.1.4, Sigmoid scores were transformed into ordinal values. Since the Hamming loss is unsuited for this evaluation, a more suitable metric is the Example Accuracy. This metric is the same as a normal accuracy metric, but each vector of predictions is consider a singular value $y$ and is either binary correct or incorrect [100]. The result across ten-fold cross-validation is $0.221,95 \%$ [0.201, 0.243]. In comparison to ten-fold cross-validation of the RFC which resulted in a score of $0.087,95 \%$ [0.086, 0.085].

$$
\text { EXAMPLE ACCURACY }=\frac{1}{N} \sum_{i=1}^{N} I\left(\hat{y}^{(i)}, y^{(i)}\right)
$$

where $\hat{y}=$ Ground truth

$y=$ Predicted score 
Table 5.1: Clinical Metrics for the Evaluation Data

\begin{tabular}{llllllll}
\hline \hline Threshold & Accuracy & Sensitivity & Specificity & \multicolumn{3}{l}{ LR+ LR- PPV NPV } \\
\hline PHQ-4 & 0.37 & $0.71(595 / 831)$ & $0.33(2350 / 6999)$ & 2.52 & 5.74 & 0.71 & 0.35 \\
\hline PHQ-2 & 0.47 & $0.57(329 / 572)$ & $0.48(3480 / 7258)$ & 1.35 & 2.25 & 0.57 & 0.47 \\
\hline GAD-2 & 0.40 & $0.71(809 / 1126)$ & $0.34(2334 / 6704)$ & 2.55 & 3.09 & 0.71 & 0.34 \\
\hline
\end{tabular}

$\mathrm{LR}+=$ positive likelihood ratio; $\mathrm{LR}-=$ negative likelihood ratio; $\mathrm{PPV}=$ positive predictive value; $\mathrm{NPV}=$ negative predictive value.

PHQ-4 $=$ Sum of all questions. Above threshold of 6 considered a positive result.

PHQ-2 $=$ Sum of first two questions. Above threshold of 3 considered a positive result.

GAD-2 $=$ Sum of last two questions. Above threshold of 3 considered a positive result.

\subsubsection{Clinical Metrics}

Sensitivity values measure how sensitive the test is correctly predicting positive cases as actually being positive. Specificity values measure how efficient the test is at correctly predicting negative (not suffering the disorder) cases from those who actually don't suffer the disorder. Both tests are good indication of the real-life implications of true negatives and false negatives. Positive and negative likelihood ratios are indications of the value of performing a test. They assess if performing the test will significantly increase the probability of knowing if an actual disorder is present or not [115]. Furthermore, positive and negative predictive values score indicate if an individual with the disorder actually has the disorder given a positive or negative test result.

The above metrics are calculated for three different scores, the PHQ-4 which is the summary of the whole assessment tool. The PHQ-2 which is the specific aspect of the tool that measures depressive symptoms and the GAD-2 which is the aspect of the tool that measures anxiety symptoms. All results are presented in Table 5.1.

\subsubsection{Limit of Agreement Tests}

To evaluate the effectiveness of our prediction system against an individual completing the PHQ-4 manually, we employ the work of the authors [116] who propose that the limit of agreement test as a reliable method of evaluating a new clinical approach compared with an established existing approach. The author propose that in cases were there is no clinical gold standard evaluation ${ }^{1}$, the most reliable method of evaluation for a new measure is an against an existing well established method. A new test would be considered "better" if it had the same prediction capabilities and overcomes some limitation or complication of the previous test. For example, if you were testing for a type of cancer with a invasive biopsy, and a new test that offered the same prediction capacity could be performed with only a smear this would be considered better overall. The limit of agreement test is proposed as a way of evaluating the difference in prediction capacity.

\footnotetext{
${ }^{1}$ An absolute diagnosis of an disorder/disease as opposite to a screening or indication of a disorder that might be proposed after a psychometric test.
} 
Table 5.2: Limit of Agreements Tests for Evaluation Data

\begin{tabular}{llll}
\hline \hline Threshold & Bias $(\bar{d})$ & ULoA & LLoA \\
\hline PHQ-4 & $-4.69[-4.78,-4.59]$ & $3.82[3.66,3.99]$ & $-13.21[-13.37,-13.04]$ \\
\hline PHQ-2 & $-2.14[-2.19,-2.10]$ & $1.79[1.72,1.87]$ & $-6.09[-6.16,-6.01]$ \\
\hline GAD-2 & $-2.54[-2.60,-2.48]$ & $2.70[2.60,2.81]$ & $-7.80[-7.90,-7.70]$ \\
\hline
\end{tabular}

$\mathrm{ULoA}=$ upper limit of agreement; LLoA = lower limit of agreement.

All confidence intervals presented at $95 \%$ level.

PHQ-4 $=$ Sum of all questions. Above threshold of 6 considered a positive result.

PHQ-2 = Sum of first two questions. Above threshold of 3 considered a positive result.

GAD-2 $=$ Sum of last two questions. Above threshold of 3 considered a positive result.

It assumes that if two tests had no difference in prediction capacity, they would predict the same scores for ever patient, regardless of which test was used. To asses this, predicted scores $y$ are subtracted from true scores $\hat{y}$ to create a $\bar{d}$ score. Again, in an ideal situation all $\bar{d}$ scores would be zero. However this is practically rarely the case, therefore the question concerns how to quantify these differences. Equation 5.2 allows the calculation of upper and lower limits of agreements (ULoA \& LLoA). These two scores provide an indication of what the upper and lower possible differences could present if using the new test.

Ideally ULoA \& LLoA should be tightly bound, indicating using the new test would produce similar results to the old one. In the case of wide bounds, to much variability may be present. Scores for the training and evaluation data are presented in Figure 5.2 .

Upper and Lower Limits of Agreement $=\bar{d} \pm 2(s)$

where $s=$ Standard deviation of the $\bar{d}$ scores

\subsection{Evaluation Participants}

A total of 154 individuals participated in the experiment between the 22th of July and the 4th of August. Participants were recruited via a variety of means, including but not limited to the mailing-distribution lists, social media and networking. No demographic details were collected. On average, users assigned to the empathy condition sent 23 messages and had an average of six words per message, while those in the non-empathy condition sent 18 with an average of five words per message.

\section{3 $\quad H_{1}$ - Hypothesis One}

The primary method of evaluation for $H_{1}$ consists of a limit of agreement tests as outlined in Section 5.1.4. The results of which are presented in Table 5.3. However, for the purpose of internal evaluation, additional metrics are reported in Table 5.4. 
Table 5.3: $H_{1}$ Limit of Agreements Tests

\begin{tabular}{llll}
\hline \hline Threshold & Bias $(\bar{d})$ & ULoA & LLoA \\
\hline PHQ-4 & $-4.94[-5.55,-4.32]$ & $2.65[1.59,3.71]$ & $-12.53[-13.59,-11.47]$ \\
\hline PHQ-2 & $-1.29[-1.63,-0.95]$ & $2.91[2.32,3.50]$ & $-5.50[-6.09,-4.91]$ \\
\hline GAD-2 & $-3.65[-4.04,-3.25]$ & $1.18[0.50,1.85]$ & $-8.47[-9.15,-7.80]$ \\
\hline
\end{tabular}

$\mathrm{ULoA}=$ upper limit of agreement; LLoA = lower limit of agreement.

All confidence intervals presented at $95 \%$ level.

PHQ-4 $=$ Sum of all questions. Above threshold of 6 considered a positive result.

PHQ-2 $=$ Sum of first two questions. Above threshold of 3 considered a positive result.

GAD-2 = Sum of last two questions. Above threshold of 3 considered a positive result.

Table 5.4: $H_{1}$ Evaluation Metrics

\begin{tabular}{ll}
\hline \hline Metric & Score \\
\hline Hamming Loss & 0.4526 \\
\hline Mean Squared Error & 4.32 \\
\hline Example Accuracy & 0.0718 \\
\hline
\end{tabular}

Furthermore, relevant clinical metrics are presented in Table 5.5. Finally, individual question confusion matrices are included in Figure 5.1.

\section{4 $H_{2}$ - Hypothesis Two}

Hypothesis 2 is composed of six specific questions (see reference Table 4.5), each evaluated on a three-point ordinal scale. One way hypothesis (less then) testing was performed for each question using a Asymptotic Linear-by-Linear Association Test (LbL) $[117,118]$. This test is derived from the family of Chi-squared $X^{2}$ tests. Given a question, it proves if there is a different distribution of responses across the three ordinal options for each of the two conditions users were placed in (empathy aware dialogue system, non-empathy aware dialogue system).

Table 5.5: $H_{1}$ Clinical Metrics

\begin{tabular}{llllllll}
\hline \hline Threshold & Accuracy & Sensitivity & Specificity & LR+ & LR- & PPV & NPV \\
\hline PHQ-4 & 0.32 & $0.86(24 / 28)$ & $0.21(26 / 125)$ & 5.99 & 0.00 & 0.09 & 0.20 \\
\hline PHQ-2 & 0.71 & $0.09(3 / 34)$ & $0.89(107 / 119)$ & 0.10 & 0.01 & 0.09 & 0.90 \\
\hline GAD-2 & 0.26 & $0.77(14 / 18)$ & $0.19(26 / 125)$ & 3.50 & 0.00 & 0.77 & 0.19 \\
\hline
\end{tabular}

$\mathrm{LR}+=$ positive likelihood ratio; $\mathrm{LR}-=$ negative likelihood ratio; $\mathrm{PPV}=$ positive predictive value; $\mathrm{NPV}=$ negative predictive value.

PHQ-4 $=$ Sum of all questions. Above threshold of 6 considered a positive result.

PHQ-2 = Sum of first two questions. Above threshold of 3 considered a positive result.

GAD-2 $=$ Sum of last two questions. Above threshold of 3 considered a positive result. 

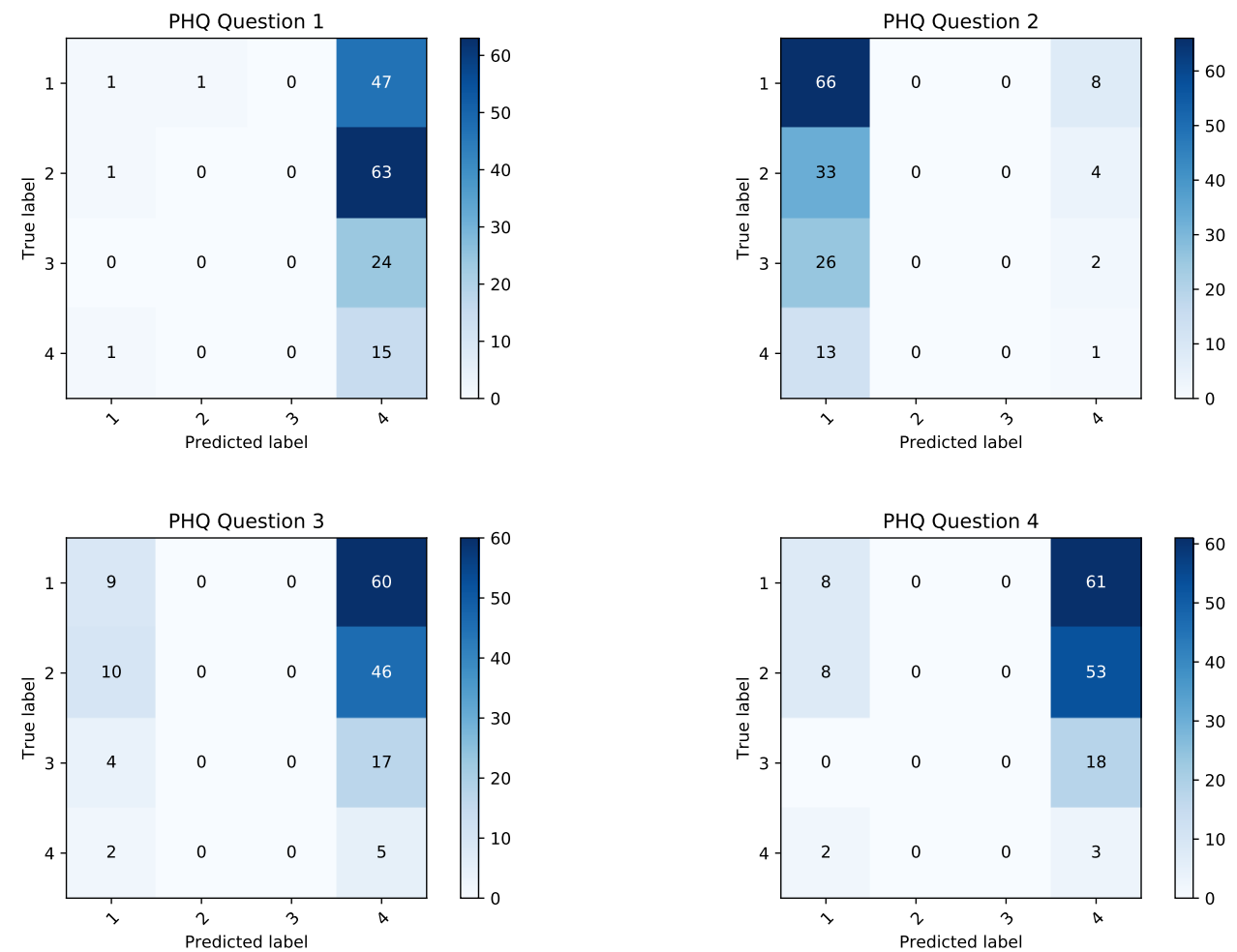

Figure 5.1: Confusion Matrix for predicted and true scores for each of the four PHQ-4 questions

However, in contrast to a Chi-squared test, an LbL incorporates the ranking order of the ordinal variables as a feature in the model. The results of an LbL test for each of the six questions are presented in Table 5.6. $z$ scores, along with $p$ values are reported for each test. Only the results for the first question are statistically significant based on a cutoff value of $<0.05$, while the results of question five are approaching significant level. Question one maintains its significant level with a two-sided hypothesis testing as well. We additionally present effect size and power level, which quantify the size of the difference between the two groups. Furthermore, Figure 5.2 presents the breakdown scores of each question divided by the condition. 

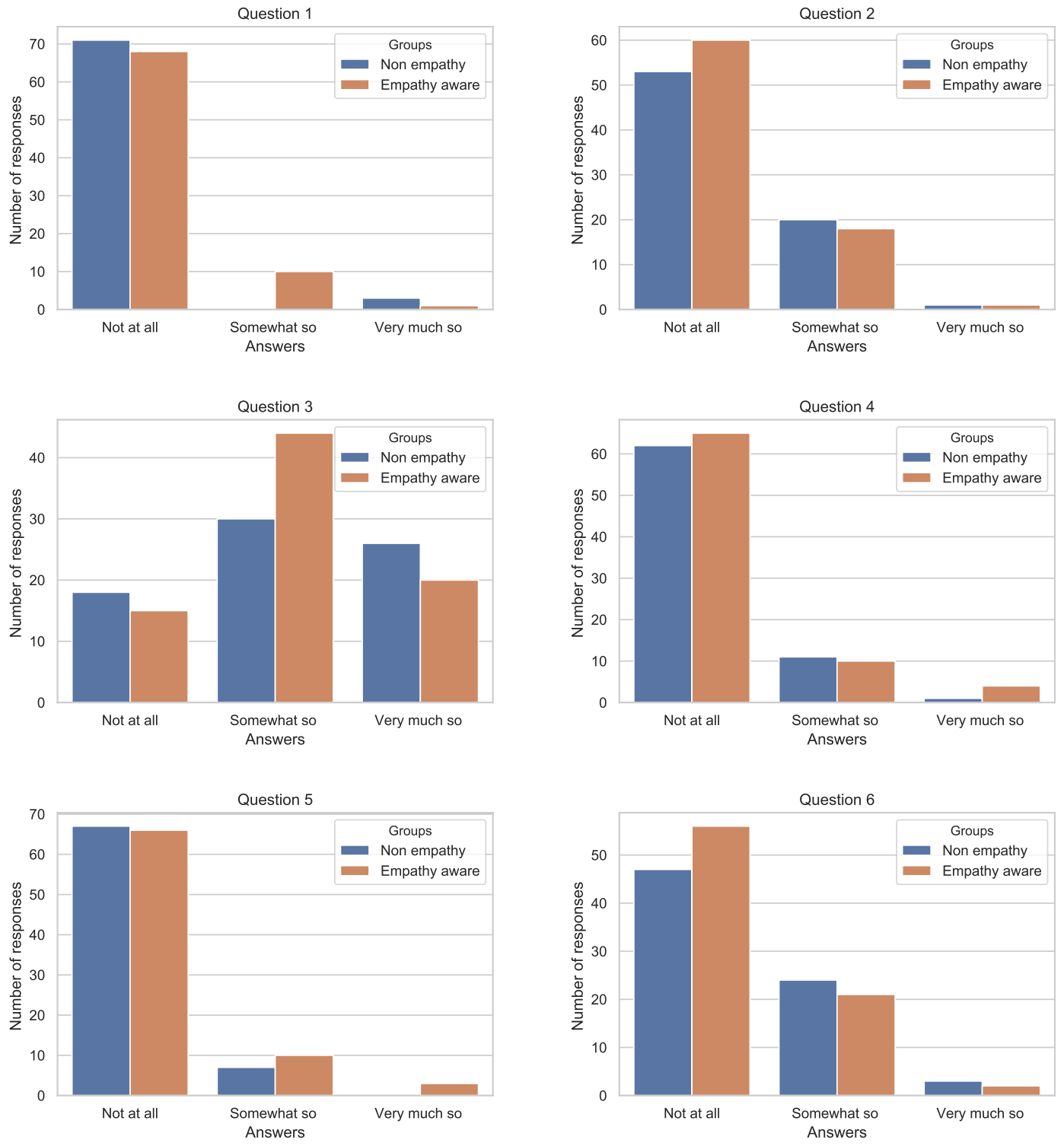

Figure 5.2: Responses for each of the six questions, grouped by assigned condition 
Table 5.6: $H_{2}$ Linear-by-Linear Association Test. ${ }^{*}=<0.05$, Given a sample size $=154 \mathrm{df}=5, \alpha=0.05$, effect size $=0.3$. We generate a power level of 0.83 and critical $x^{2}$ of 3.84

\begin{tabular}{|c|c|c|}
\hline Question & $z$ & $p$ \\
\hline $\begin{array}{l}\text { 1. Did the responses from the system show understanding } \\
\text { of your feeling/experiences? }\end{array}$ & -2.153 & $0.015^{*}$ \\
\hline $\begin{array}{l}\text { 2. Where the responses seem appropriate to the conver- } \\
\text { sation? (Were they on topic) }\end{array}$ & 0.572 & 0.716 \\
\hline $\begin{array}{l}\text { 3. Could you understand the responses? (Did the lan- } \\
\text { guage seem accurate) }\end{array}$ & 0.386 & 0.650 \\
\hline 4. Did you feel trust toward the system? & -0.674 & 0.249 \\
\hline 5. Did you feel the system understood your emotions? & -1.627 & 0.051 \\
\hline 6. Did you feel the system had emotions? & 1.008 & 0.843 \\
\hline
\end{tabular}




\section{6}

\section{Conclusion}

\subsection{Overview}

The content of this thesis crosses two domains, that of clinical psychology and natural language processing (with the use of applied machine learning). We begin by establishing that depression and anxiety are highly prevalent illnesses worldwide and the current approaches to screening individuals for these disorders suffer from a number of inherent limitations. One such limitation we propose to address is the reliance on individuals completing psychometric screening questionnaires.

Based on an existing body of work, we propose a hypothesis $\left(H_{1}\right)$ that linguistic features in a text could be employed to predict how an individual would complete the PHQ-4 psychometric questionnaire. Going forward from this, we established that research has shown that social desirability can impact the honesty of responses in patient-doctor settings. Dialogue systems have been demonstrated as a potential approach to overcome this issue.

We further propose that the linguistic features mentioned above can be detected from a conversation with a dialogue system. However, to achieve this in a clinical setting. There is a requirement that these system be able to appropriately respond to individuals. In order to do so, a level of empathy is required to be demonstrated by the system.

Our work, therefore, begins by reviewing previous approaches that have been employed to achieve this. Given our interpretation of the literature, we propose the second hypothesis $\left(\mathrm{H}_{2}\right)$, that encoding emojis into a sequence-to-sequence neural approach will allow a dialogue system to be able to display better emotional understanding as evaluated by users in a randomized trial.

To develop the machine learning classifier, we began by employing the DIAC-WOZ as training data. The dataset contains chatlogs from interviews with volunteers, along with matched PHQ scores taken during the interview. Three types of features we extracted from this data, word embeddings, psycholinguistic scores, and psychometric similarity scores.

We employed a deep neural network architecture to predict PHQ-4 score in a multidimensional output form. The hyperparameters of the network were optimized with the use of an evolutionary algorithm. The results of the training stage of the model are presented in Section 5.1. Where we demonstrated that our model performed better than random baseline in all cases. Furthermore, our model outperformed a simpler random forest classifier which validated the use of a deep neural network versus a simpler more interpretable model. 
Two dialogue systems were developed using bidirectional LSTMs on sentence aligned datasets (OpenSubtitles). One of these systems has no specific approach to model empathy, however, the other system predates each training sequence with emojis tokens from both the source and target text sequence.

To evaluate both hypotheses, a public-facing experiment was launched. This experiment was approved by the university ethics committee and had an approved data protection impact assessment completed. 154 participants were recruited across a two week period. These participants were asked to have a short conversation with one of the two dialogue systems (random allocation). Following this, participants completed six Likert scale questions on how they felt the system modelled their emotions.

At the same time, participants were asked to complete the PHQ-4 psychometric questionnaire. Chatlogs from the conversation were stored and analysed using our prediction algorithm trained above. The predicted PHQ-4 scores were then compared against manually completed PHQ-4 scores using a limit of agreement tests.

\subsection{Ethical Discussion}

The fundamental question raised in this thesis concerns if predictive analytics can be achieved for a mental health disorder through extracting linguistic features. This question raises a range of ethical concerns and considerations. Public perception of predictive health analytics varies greatly across stakeholders, within the United Kingdoms survey reports estimate that $63 \%$ of adult are uncomfortable with allowing AI systems replacing doctors and nurses in tasks they usually perform [119]. However, up to $83 \%$ of medical students agree that AI systems will positively improve their workplace [120]. The final stakeholder group, state decision makers and healthcare organisations report confident that it will improve medicine, but roughly half of them think it will produce fatal errors, will not work properly, and will not meet currently hyped expectations [120].

Noting this disintegration, it is clear that greater consideration from all stakeholder need to be achieved. The authors of [121] propose a number of questions that can be asked to better achieve consensus. Firstly, a clinician must identify an area that has a substantial need for improvement, and which machine learning in medicine (MLm) can reasonably be applied to. This is not always as simple as it would appear, at a minimum, there is generally a requirement that some form of theoretical concept would explain why a given set of data would allow predictive qualities of a disorder [8].

Secondly, the authors of $[121,122]$ propose that the risk associated with the predictive system is ethical given the underlying risk of not using the system. A number of inherent potential risks should always be considered when using MLm systems. Firstly, it has been repeatedly demonstrated in the literature that ML methods are poor at generalizing outside of trained data [76]. Within this thesis, our training data from the DIAC-WOZ was collected from a sample size within the United States, it is therefore reasonable to assume that the linguistic pattern our model considers important may be heavily influenced by this limited sample size. We have no way of knowing if our system would perform equally well for English speakers who have 
learnt outside of the colloquial language pattern of the area.

Furthermore, this could disproportionately bias certain types of predictions for certain groups of people. We do not have demographic variables for the participants who supplied our training data. However, a core facet of medical profession is equal treatment to all groups of people. Simply ignoring demographic variables in our training data does not necessarily create an unbiased model, but rather we should be aware of limitations in our training data and consider manually adjusting this to ensure a fair and accurate system that is neutral to concepts such as gender, age, race, income etc.

Within the context of our this thesis, we consider that bias is a key area to be addressed if this research continue forwards. Effort would need to be made to ensure the training data matches the population it will applied to in terms of lingustic patterns, but also, that reasonable effort has been made to ensure fairness across different personal traits were appropriate.

Secondly, a disadvantage of ML algorithms is often their propensity to a "black box" nature. Where by, a medical professional could reasonability explain to a patient how given their symptoms they have reached a certain conclusion. With deep learning models, it is both technically hard to explain to a patient how certain feature effect the probability of an outcome, but also it is sometimes computationally impossible to explain how these features interact within a algorithm. Although an increasing effort has been made in recent years to better develop computational methods that can allow for understanding of the processing occurring within these algorithms [123].

This has led to an ongoing disintegration between the latest version of the European data protection regulations and current deep learning approaches. The GDPR framework considers interpretability of decision making to be a core principle of the framework. This is directly at odds if there is an inability for any of these methods to provide interpretive results. This issue is not limited to MLm, but relevant in any application were deep learning is involved in automated decision making [124]. Finally, the issue of informed consent becomes relevant in these situations. In a medical setting, informed consent of medical treatment has always been a core and required aspect of clinical and research practice [125]. Many medical practices require consent regardless, it would be quite hard to take blood from someone without their knowledge. However, the nature of predictive health from linguistic pattern is considerability more subtle. Individuals may not be aware their written or verbal communication is being processed in this manner, since it's doesn't effect them. Within a professional medical setting, it would be unethical to perform any such process without the informed consent of a patient.

However, private companies do not need to uphold to such ethical standards. Indications have already emerged that certain companies have began to experiment with predictive or inferential analysis of health information from linguistic pattern. Examples include the social media giant Facebook [126]. The GDPR framework prevents private companies performing this type data analysts without consent, but other legal jurisdictions such as the United States and Asia do not have this level of legal framework [126].

The questions proposed above from the authors of [121] are a important starting 
ground for any work in this area. Given our interpretation above, it is clear that this work is far from being ethically implemented in practice. Despite this, there is still little legal requirement for these types of system to meet any ethical standards. Although this is likely to change as the public and law maker knowledge of these systems increases.

Within both the context of a student master thesis, and of applied data science. It is clear that work in this field has core ethical considerations. Which may influence how future data scientists are trained, and how research institutes that previously haven't had to consider ethics adapt and support this.

\subsection{Research Questions Discussion}

Initial results presented in Section 5.1 demonstrate that the approach we employed in training our machine learning classifier outperformed random baseline allocation by 0.11 when measured using the Hamming loss criteria. Furthermore, our model outperformed a trained random forest approach by 0.14 when evaluated using an accuracy score. This demonstrates that although there was a trade-off in the interpretability by employing a multilayered architecture, in this case, our proposed approach was better able to model the interconnectivity of the multidimensional data.

In addition to simply interpreting the predictive ability of the model, additional metrics from the clinical domain can be employed to examine the implications of these results in practical settings. Within the literature, PHQ-4 scores are generally interpreted in three ways. Firstly, the combination of all four questions can be combined and the resulting value can be divided on a threshold level of $<6$ or $=>6$. Secondly, the first two questions on the PHQ-4 are designed to assess the primary symptoms of anxiety, and represent the GAD-2 questionnaire. Therefore, we can consider the sum of these questions to be able to be cut on a threshold of $<3$ or $=>3$. Finally, the same applies to the last two questions on the PHQ-4, which represent the PHQ-2 questionnaire. We use these three scales, either in a binary or ordinal form to evaluate the model.

When we evaluate the model on the evaluation data from the DAIC-WOZ, we see reasonable sensitivity scores, but poor specificity indicating over prediction of the disorder. For the evaluation of the experimental data (Table 5.4), the same results are seen with the expectation of the PHQ-2. Which has a very low sensitivity and very high specificity score. Based on the confusion matrix plots seen in Figure 5.1 this is likely due to the model almost exclusively predicting a score of 3 for all participants.

For the evaluation data, in all cases, our positive likelihood ratio $(\mathrm{LR}+)$ scores indicate that there is a slight to medium effectiveness in performing the test in order to detect a positive result. However, these scores are quite poor for LR- when the outcome of the test is negative. This is linked to the poor specificity scores we mentioned early. LR+ are notably higher for the experimental data, which is a positive sign, however, it is counteracted by extremely low LR-. Indicating the test is likely of no use in predicting a negative outcome.

For the evaluation data, PPV and NPV scores are as reasonable as to be expected 
given the previously mentioned scores. However, for the experimental data, PPV scores are very low for the PHQ-4 and PHQ-2 indicating a low likelihood of predicting a positive scores, however, given higher sensitivity scores for the PHQ-4 this is not an issue, and we have already mentioned the noted issue with the PHQ-2. Results for the GAD-2 and NPV scores are as to be expected given the previously reported results.

Our main method of evaluation of $H_{1}$ was employed using a limit of agreement tests. The results are presented in Table 5.3 and compared with the evaluation data in Table 5.2. In this case, our focus is mostly on the bias $(\bar{d})$ score, which indicates the difference between the predicted score and the actual score. For all cases, our target is the minimization of this score.

For the evaluation data, all $\bar{d}$ scores are high, indicating a high difference between what the model predicts and ground truth. With the expectation of the PHQ-2, all these scores are similar for the experimental data. This indicates that the model is consistent in its performance on evaluation and unseen data. The PHQ-2 would appear to perform better, with a tighter limit bound and smaller bias term. As mentioned previously, it is subjective as to what is an acceptable bias term and limit bound. However, scores for the PHQ-4 and GAD-2 are high, indicating on that on average a predicted value is 5 points different for the PHQ- 4 and 4 points different for the GAD-2.

Figure 5.1 shows the confusion matrices for each of the four questions on the PHQ-4. We see here that in most cases the model predicts scores of either 0 or 3 for each question, indicating quite poor performance. For the purpose of a screening test, we consider both of these scores as being too high. Therefore, for predicting PHQ-4 and GAD-2 scores, we accept the first Null Hypothesis. However, the bias term for the PHQ-2 is only 1. We consider this a reasonable score for the prediction model. Hence, we reject the null Hypothesis for this question.

To evaluate the effectiveness of our empathy approach, we employed six Likert style questions. The difference between how users answered these questions was assessed using a linear-by-linear association test. Only in the case of the first question did the two conditions (empathy, non-empathy) significantly differ in their answering. Question five approaches a significant level. The results seen from question one indicate that our empathic approach displayed a better understanding of feelings and experiences compared with the non-empathic condition.

Given that the five other questions did not differ, we can establish that although the approach made some significant improvement compared to the baseline, there is still a considerable space for improvement. Its important to note however that question five (if you felt the system understood your emotions) was approaching significant with a one-way test. This is an important consideration, given that a key feature of empathy is to be able to give an individual the idea that you are recognizing their emotions. Given the above results, we can reject one of six sub Null Hypothesis for $\mathrm{H}_{2}$. 


\subsection{Considerations and Future Work}

In reflection on this work, we note two limitations and a challenge. The first of which; is that it has been repeatedly demonstrated in the NLP literature that different domain or styles of text can affect the generalizability of a model. We propose that the relativity poor performance of our classification model on the experimental data was due to the limited amount of information that could be extracted from the chatlogs. Our training data from the DIAC-WOZ had an average of 168 messages per user, while our experimental data had 18 messages per user.

While considering the importance of volunteers time, a higher threshold for the number of messages that we required participants to talk to our system should have been set. Furthermore, although we do not rule out using dialogue systems for this approach, we propose that the amount of information that needs to be conveyed within these conversations is higher than initially thought.

The second limitation is with regards to the experimental design. A limited period of internal user testing before the experiment was launched resulted in a issue occurring while it was open to the public. Participants who completed the experiment on a mobile device could not move forward through the whole experiment. The program would not allow them to pass the dialogue system stage. This resulted in roughly an additional 80 volunteers attempting to take part in the study but not being able to. If this issue had been fixed, a higher sample size could have achieved. This limitation shows the importance of user testing.

As the fields of applied data science and medicine converge more, new questions and considerations need to come to the forefront. The work presented here has both practical and theoretical ethical considerations. A considerable amount of time within the project was spent on submitting an ethical application to the university board. This is one of the first times the board would have received a study of this nature, and considerations need to be made for how these types of study differ to classical medical work, and how best to assess them. On a theoretical level, considerations need to be made for the long term implication of this work. Although this work was done in a good faint, and with an aim to improve the lives of people, there is a knowledge that it could be potentially used to screen and harm people in the future.

Given the above limitations and challenges, we examine our overall contribution to the literature. To begin with, our work demonstrates an ability to develop deep learning models to predict PHQ-4 scores given textual data using psycholinguistics feature. Compared with previous work in the area $[10,75]$ machine learning can be used to model outputs that are easily interpretable by both machine learning and medicine domain experts. Our work is the first to propose that psychometric similarity scores might be a useful feature within this domain going forward. Additional work going forward needs to examine how to improve the generalizability of this work to allow it be applied across different domains.

Regarding our second Hypothesis, our contribution to the literature is firstly, that emoji tokens are better representations of emotions compared to annotations of Elkman emotional categories. Secondly, we demonstrate that predating each dialogue utterance with both source and target emotions is efficient in improving empathy 
appearance. We propose that future work in this area would investigate how the diversity of emojis is related to performance. Not ignoring that considerable work is still required on developing neural generative models that can hold a reasonable conversation. 


\section{Bibliography}

[1] Albert Haque et al. "Measuring Depression Symptom Severity from Spoken Language and 3D Facial Expressions". 2018.

[2] World Health Organization. Depression Fact Sheet. Tech. rep. 2018.

[3] Eric J Topol. "High-performance medicine: the convergence of human and artificial intelligence." In: Nature medicine 25.1 (2019), pp. 44-56.

[4] Klaus P. Ebmeier, Claire Donaghey, and J. Douglas Steele. "Recent developments and current controversies in depression". In: Lancet 367.9505 (2006), pp. $153-167$.

[5] N Sartorius and B Oston. "Depression comorbid with anxiety : results from the WHO study on psychological disorders in primary health care . Publication Types, MeSH Terms , Substances". In: 168.30 (1996), p. 8864147.

[6] Hans Ulrich Wittchen. "Generalized anxiety disorder: Prevalence, burden, and cost to society". In: Depression and Anxiety 16.4 (2002), pp. 162-171.

[7] American Psychiatric Association. Diagnostic and Statistical Manual of Mental Disorders, 5th Edition: DSM-5. American Psychiatric Pub, 2013, p. 947.

[8] Fionn Delahunty, Ian D. Wood, and Mihael Arcan. "First insights on a passive major depressive disorder prediction system with incorporated conversational chatbot". In: CEUR Workshop Proceedings. Vol. 2259. 2018, pp. 327-338.

[9] Munmun De Choudhury et al. "Discovering Shifts to Suicidal Ideation from Mental Health Content in Social Media". In: Proceedings of the 2016 CHI Conference on Human Factors in Computing Systems - CHI '16 (2016), pp. 2098-2110.

[10] David E Losada and Fabio Crestani. "A Test Collection for Research on Depression and Language Use CLEF 2016, Évora (Portugal)". In: Experimental IR Meets Multilinguality, Multimodality, and Interaction (2016), pp. 28-29.

[11] Kyo Joong Oh et al. "A chatbot for psychiatric counseling in mental healthcare service based on emotional dialogue analysis and sentence generation". In: Proceedings - 18th IEEE International Conference on Mobile Data Management, MDM 2017 (2017), pp. 371-376.

[12] Magalie Ochs, David Sadek, and Catherine Pelachaud. "A formal model of emotions for an empathic rational dialog agent". In: Autonomous Agents and Multi-Agent Systems 24.3 (2012), pp. 410-440.

[13] Juan Martínez-Miranda. "Embodied Conversational Agents for the Detection and Prevention of Suicidal Behaviour: Current Applications and Open Challenges". In: Journal of Medical Systems 41.9 (2017). 
[14] Timothy Bickmore and Toni Giorgino. "Health dialog systems for patients and consumers". In: Journal of biomedical informatics 39.5 (2006), pp. 556571.

[15] Timothy W Bickmore et al. "Improving access to online health information with conversational agents: a randomized controlled experiment". In: Journal of medical Internet research 18.1 (2016), e1.

[16] Adam S Miner et al. "Smartphone-based conversational agents and responses to questions about mental health, interpersonal violence, and physical health". In: JAMA internal medicine 176.5 (2016), pp. 619-625.

[17] Kathy A. Stepien and Amy Baernstein. "Educating for empathy: A review". In: Journal of General Internal Medicine 21.5 (2006), pp. 524-530.

[18] Hannah Rashkin et al. "I Know the Feeling: Learning to Converse with Empathy". In: 1994 (2018), pp. 1-13.

[19] Kathleen Kara Fitzpatrick, Alison Darcy, and Molly Vierhile. "Delivering cognitive behavior therapy to young adults with symptoms of depression and anxiety using a fully automated conversational agent (Woebot): a randomized controlled trial". In: JMIR mental health 4.2 (2017), e19.

[20] Tim Althoff, Kevin Clark, and Jure Leskovec. "Large-scale Analysis of Counseling Conversations: An Application of Natural Language Processing to Mental Health". In: 4 (2016), pp. 463-476.

[21] Aaron T. Beck. "Cognitive therapy: A 30-year retrospective". In: American Psychologist 46.4 (1991), pp. 368-375.

[22] John R.Z. Abela and David U. D'Alessandro. "Beck's cognitive theory of depression: A test of the diathesis-stress and causal mediation components". In: British Journal of Clinical Psychology 41.2 (2002), pp. 111-128.

[23] David A. Clark, Robert A. Steer, and Aaron T. Beck. "Common and Specific Dimensions of Self-Reported Anxiety and Depression: Implications for the Cognitive and Tripartite Models". In: Journal of Abnormal Psychology 103.4 (1994), pp. 645-654.

[24] Aaron Beck. "Beck Depression Inventory". In: (1996).

[25] Lee Anna Clark and David Watson. "Tripartite Model of Anxiety and Depression: Psychometric Evidence and Taxonomic Implications". In: Journal of Abnormal Psychology 100.3 (1991), pp. 316-336.

[26] Alexandra Martin et al. "Validity of the brief patient health questionnaire mood scale (PHQ-9) in the general population". In: General hospital psychiatry 28.1 (2006), pp. 71-77.

[27] Kurt Kroenke et al. "An ultra-brief screening scale for anxiety and depression: the PHQ-4". In: Psychosomatics 50.6 (2009), pp. 613-621.

[28] Adrian Furnham. "Response bias, social desirability and dissimulation". In: Personality and individual differences 7.3 (1986), pp. 385-400.

[29] Claire Henderson et al. "Mental health-related stigma in health care and mental health-care settings". In: The Lancet Psychiatry 1.6 (2014), pp. 467482.

[30] Ryan M. Schuetzler et al. "The Influence of Conversational Agents on Socially Desirable Responding". In: Proceedings of the Hawaii International Conference on System Sciences (HICSS) 9 (2018), pp. 283-292. 
[31] Robert Johansson and Gerhard Andersson. "Internet-based psychological treatments for depression". In: Expert Review of Neurotherapeutics 12.7 (2012), pp. 861-870.

[32] Simon Provoost et al. "Embodied conversational agents in clinical psychology: a scoping review". In: Journal of medical Internet research 19.5 (2017), e151.

[33] Adam S Miner, Clinical Excellence, and Behavioral Sciences. "Talking to Machines About Personal Mental Health Problems". In: 94305 (2019).

[34] Pascale Fung et al. "Empathetic Dialog Systems". In: The International Conference on Language Resources and Evaluation. European Language Resources Association. 2018.

[35] Jochanan Benbassat and Reuben Baumal. "What is empathy, and how can it be promoted during clinical clerkships?" In: Academic Medicine 79.9 (2004), pp. 832-839.

[36] Jodi Halpern. "What is clinical empathy?" In: Journal of general internal medicine 18.8 (2003), pp. 670-674.

[37] Paul S Bellet and Michael J Maloney. "The importance of empathy as an interviewing skill in medicine". In: Jama 266.13 (1991), pp. 1831-1832.

[38] Stewart W Mercer and William J Reynolds. "Empathy and quality of care." In: Br J Gen Pract 52.Suppl (2002), S9-12.

[39] Christine L. Cox et al. "The balance between feeling and knowing: Affective and cognitive empathy are reflected in the brain's intrinsic functional dynamics". In: Social Cognitive and Affective Neuroscience 7.6 (2012), pp. 727737.

[40] Courtney Corley and Rada Mihalcea. "Measuring the semantic similarity of texts". In: June (2010), pp. 13-18.

[41] Renate LEP Reniers et al. "The QCAE: A questionnaire of cognitive and affective empathy". In: Journal of personality assessment 93.1 (2011), pp. 8495.

[42] Simone G. Shamay-Tsoory, Judith Aharon-Peretz, and Daniella Perry. "Two systems for empathy: A double dissociation between emotional and cognitive empathy in inferior frontal gyrus versus ventromedial prefrontal lesions". In: Brain 132.3 (2009), pp. 617-627.

[43] SG Shamay-Tsoory et al. "Impairment in cognitive and affective empathy in patients with brain lesions: anatomical and cognitive correlates". In: Journal of clinical and experimental neuropsychology 26.8 (2004), pp. 1113-1127.

[44] Peter K Jonason and Laura Krause. "The emotional deficits associated with the Dark Triad traits: Cognitive empathy, affective empathy, and alexithymia". In: Personality and Individual Differences 55.5 (2013), pp. 532-537.

[45] F.D.A. Proposed Regulatory Framework for Modifications to Artificial Intelligence/Machine Learning (AI/ML)-Based Software as a Medical Device (SaMD)-Discussion Paper and Request for Feedback. Tech. rep. Food and Drug Administration (US), 2019, pp. 1-20.

[46] Colin G. Walsh, Jessica D. Ribeiro, and Joseph C. Franklin. "Predicting Risk of Suicide Attempts Over Time Through Machine Learning". In: Clinical Psychological Science 5.3 (2017), pp. 457-469. 
[47] Jonathan Gratch et al. "The Distress Analysis Interview Corpus of human and computer interviews". In: Lrec (2014), pp. 3123-3128.

[48] Cheongjae Lee et al. "Example-based dialog modeling for practical multidomain dialog system". In: Speech Communication 51.5 (2009), pp. 466-484.

[49] Robert P Schumaker et al. "Evaluating mass knowledge acquisition using the alice chatterbot: The az-alice dialog system". In: International journal of human-computer studies 64.11 (2006), pp. 1132-1140.

[50] Hongshen Chen et al. "A survey on dialogue systems: Recent advances and new frontiers". In: ACM SIGKDD Explorations Newsletter 19.2 (2017), pp. 2535.

[51] Tomáš Mikolov et al. "Recurrent neural network based language model". In: Eleventh annual conference of the international speech communication association. 2010.

[52] Sepp Hochreiter and Jürgen Schmidhuber. "Long short-term memory". In: Neural computation 9.8 (1997), pp. 1735-1780.

[53] Mike Schuster and Kuldip K Paliwal. "Bidirectional recurrent neural networks". In: IEEE Transactions on Signal Processing 45.11 (1997), pp. 26732681.

[54] Rico Sennrich, Barry Haddow, and Alexandra Birch. "Neural machine translation of rare words with subword units". In: arXiv preprint arXiv:1508.07909 (2015).

[55] Zellig S Harris. "Distributional structure". In: Papers in structural and transformational linguistics. Springer, 1970, pp. 775-794.

[56] Quoc V. Le and Tomas Mikolov. "Distributed Representations of Sentences and Documents". In: 32 (2014).

[57] Jey Han Lau and Timothy Baldwin. "An empirical evaluation of doc2vec with practical insights into document embedding generation". In: arXiv preprint arXiv:160\%.05368 (2016).

[58] Ashish Vaswani et al. "Attention is all you need". In: Advances in neural information processing systems. 2017, pp. 5998-6008.

[59] R Simon and David G Altman. "Statistical aspects of prognostic factor studies in oncology." In: British journal of cancer 69.6 (1994), p. 979.

[60] Mohammed Al-mosaiwi and Tom Johnstone. "In an Absolute State : Elevated Use of Absolutist Words Is a Marker Specific to Anxiety, Depression, and Suicidal Ideation". In: (2018).

[61] Stefan G Hofmann et al. "Linguistic correlates of social anxiety disorder". In: Cognition \& emotion 26.4 (2012), pp. 720-726.

[62] Cindy Chung and James Pennebaker. "The Psychological Functions of Function Words". In: (2007), pp. 343-359.

[63] James W Pennebaker, Matthias R Mehl, and Kate G Niederhoffer. "Psychological aspects of natural language. use: our words, our selves." In: (2003).

[64] Stephanie Rude, Eva-maria Gortner, and James Pennebaker. "Language use of depressed and depression- vulnerable college students college students". In: 9931 (2010).

[65] G Miller. The Science of Words. New York: Sci. Am. 1995. 
[66] Yla R Tausczik and James W Pennebaker. "The Psychological Meaning of Words : LIWC and Computerized Text Analysis Methods". In: (2010).

[67] Yen-Hao Huang, Lin-Hung Wei, and Yi-Shin Chen. "Detection of the Prodromal Phase of Bipolar Disorder from Psychological and Phonological Aspects in Social Media". In: (2017).

[68] Faneva Ramiandrisoa and Farah Benamara. "IRIT at e-Risk 2018". In: ERisk workshop. 2018, pp. 367-377.

[69] John Pestian et al. "Suicide Note Classification Using Natural Language Processing: A Content Analysis." In: Biomedical informatics insights 2010.3 (2010), pp. 19-28.

[70] Judit Fekete, Viktor Voros, and Author Contributions. "Linguistic Research on Specific Features of Suicidal Communication — Past, Present, and Future". In: 2002 (2018).

[71] Georgia Tech, Atlanta Ga, and Mark Dredze. "Discovering shifts to suicide ideation from mental h". In: (2017).

[72] Colin G Walsh, Jessica D Ribeiro, and Joseph C Franklin. "Predicting Risk of Suicide Attempts Over Time Through Machine Learning". In: (2017).

[73] Munmun De Choudhury, Scott Counts, and Eric Horvitz. "Social media as a measurement tool of depression in populations". In: Proceedings of the 5th Annual ACM Web Science Conference on - WebSci '13 (2013), pp. 47-56.

[74] Munmun De Choudhury and Sushovan De. "Mental Health Discourse on reddit: Self-Disclosure, Social Support, and Anonymity". In: Proceedings of the Eight International AAAI Conference on Weblogs and Social Media (2014), pp. $71-80$.

[75] Maxim Stankevich et al. "Feature Engineering for Depression Detection in Social Media". In: Icpram (2018), pp. 426-431.

[76] Fionn Delahunty, Robert Johansson, and Mihael Arcan. "Passive Diagnosis incorporating the PHQ-4 for Depression and Anxiety". In: Social Media Mining for Health Applications Workshop. 2019, pp. 367-377. DOI: 10.13025 / ee3h-yz88.

[77] David E Losada, Fabio Crestani, and Javier Parapar. "CLEF 2017 eRisk Overview: Early Risk Prediction on the Internet: Experimental Foundations." In: CLEF (Working Notes). 2017.

[78] David E Losada, Fabio Crestani, and Javier Parapar. "Overview of eRisk: Early Risk Prediction on the Internet". In: International Conference of the Cross-Language Evaluation Forum for European Languages. Springer. 2018, pp. 343-361.

[79] Marcel Trotzek, Sven Koitka, and Christoph M Friedrich. "Word Embeddings and Linguistic Metadata at the CLEF 2018 Tasks for Early Detection of Depression and Anorexia". In: ().

[80] Marcel Trotzek, Sven Koitka, and Christoph M. Friedrich. "Utilizing Neural Networks and Linguistic Metadata for Early Detection of Depression Indications in Text Sequences". In: (2018).

[81] Faneva Ramiandrisoa et al. "IRIT at e-Risk 2018". In: E-Risk workshop. 2018, pp. $367-377$. 
[82] James H Martin and Daniel Jurafsky. Speech and language processing: An introduction to natural language processing, computational linguistics, and speech recognition. Pearson/Prentice Hall Upper Saddle River, 2009.

[83] Xin Rong. "Word2vec parameter learning explained". In: arXiv preprint arXiv:1411.2738 (2014).

[84] Elena Tutubalina and Sergey Nikolenko. "Exploring convolutional neural networks and topic models for user profiling from drug reviews". In: Multimedia Tools and Applications 77.4 (2018), pp. 4791-4809.

[85] Zhilin Yang, Ruslan Salakhutdinov, and William W Cohen. "Transfer learning for sequence tagging with hierarchical recurrent networks". In: arXiv preprint arXiv:1703.06345 (2017).

[86] Matthew E Peters et al. "Deep contextualized word representations". In: arXiv preprint arXiv:1802.05365 (2018).

[87] Jacob Devlin et al. "BERT: Pre-training of Deep Bidirectional Transformers for Language Understanding". In: arXiv preprint arXiv:1810.04805 (2018).

[88] Victor Leiva and Ana Freire. "Towards suicide prevention: early detection of depression on social media". In: International Conference on Internet Science. Springer. 2017, pp. 428-436.

[89] Farig Sadeque, Dongfang Xu, and Steven Bethard. "Measuring the latency of depression detection in social media". In: Proceedings of the Eleventh ACM International Conference on Web Search and Data Mining. ACM. 2018, pp. $495-503$.

[90] Nabiha Asghar et al. "Affective neural response generation". In: Lecture Notes in Computer Science (including subseries Lecture Notes in Artificial Intelligence and Lecture Notes in Bioinformatics) 10772 LNCS (2018), pp. 154166.

[91] Xiao Sun, Xiaoqi Peng, and Shuai Ding. "Emotional Human-Machine Conversation Generation Based on Long Short-Term Memory". In: Cognitive Computation 10.3 (2018), pp. 389-397.

[92] Marcin Skowron et al. "The good, the bad and the neutral: Affective profile in dialog system-user communication". In: Lecture Notes in Computer Science (including subseries Lecture Notes in Artificial Intelligence and Lecture Notes in Bioinformatics) 6974 LNCS.PART 1 (2011), pp. 337-346.

[93] Robert Johansson et al. "Depression, anxiety and their comorbidity in the Swedish general population: point prevalence and the effect on health-related quality of life". In: PeerJ 1 (2013), e98.

[94] Melissa McTernan and Shelley A Blozis. "Longitudinal models for ordinal data with many zeros and varying numbers of response categories". In: Structural Equation Modeling: A Multidisciplinary Journal 22.2 (2015), pp. 216226.

[95] Kevin L Delucchi and Alan Bostrom. "Methods for analysis of skewed data distributions in psychiatric clinical studies: working with many zero values". In: American Journal of Psychiatry 161.7 (2004), pp. 1159-1168.

[96] Daniel Cer et al. "Universal sentence encoder". In: arXiv preprint arXiv:1803.11175 (2018). 
[97] Rada Mihalcea, Courtney Corley, Carlo Strapparava, et al. "Corpus-based and knowledge-based measures of text semantic similarity". In: AAAI. Vol. 6. 2006, pp. 775-780.

[98] Yuhua Li, Zuhair A Bandar, and David McLean. "An approach for measuring semantic similarity between words using multiple information sources". In: IEEE Transactions on knowledge and data engineering 15.4 (2003), pp. 871882.

[99] Jürgen Schmidhuber. "Deep learning in neural networks: An overview". In: Neural networks 61 (2015), pp. 85-117.

[100] Jesse Read, Concha Bielza, and Pedro Larrañaga. "Multi-dimensional classification with super-classes". In: IEEE Transactions on knowledge and data engineering 26.7 (2014), pp. 1720-1733.

[101] Jinseok Nam et al. "Large-scale multi-label text classification - Revisiting neural networks". In: Lecture Notes in Computer Science (including subseries Lecture Notes in Artificial Intelligence and Lecture Notes in Bioinformatics) 8725 LNAI.PART 2 (2014), pp. 437-452.

[102] Min-Ling Zhang and Zhi-Hua Zhou. "A review on multi-label learning algorithms". In: IEEE transactions on knowledge and data engineering 26.8 (2014), pp. 1819-1837.

[103] Eneldo Loza Mencıa and Johannes Fürnkranz. "Pairwise learning of multilabel classifications with perceptrons". In: IEEE International Joint Conference on Neural Networks. 2008.

[104] Ouadie Gharroudi, Haytham Elghazel, and Alex Aussem. "A comparison of multi-label feature selection methods using the random forest paradigm". In: Canadian conference on artificial intelligence. Springer. 2014, pp. 95-106.

[105] Risto Miikkulainen et al. "Evolving deep neural networks". In: Artificial Intelligence in the Age of Neural Networks and Brain Computing. Elsevier, 2019, pp. 293-312.

[106] Frauke Friedrichs and Christian Igel. "Evolutionary tuning of multiple SVM parameters". In: Neurocomputing 64 (2005), pp. 107-117.

[107] Alan S Cowen and Dacher Keltner. "Self-report captures 27 distinct categories of emotion bridged by continuous gradients". In: Proceedings of the National Academy of Sciences 114.38 (2017), E7900-E7909.

[108] Bjarke Felbo et al. "Using millions of emoji occurrences to learn any-domain representations for detecting sentiment, emotion and sarcasm". In: arXiv preprint arXiv:1708.00524 (2017).

[109] Ian D. Wood and Sebastian Ruder. "Emoji as Emotion Tags for Tweets". In: Proceedings of LREC 2016 Workshop, Emotion and Sentiment Analysis (2016), pp. 76-79.

[110] Thanh-Le Ha, Jan Niehues, and Alexander Waibel. "Toward multilingual neural machine translation with universal encoder and decoder". In: arXiv preprint arXiv:1611.04798 (2016).

[111] Pierre Lison and Jörg Tiedemann. "Opensubtitles2016: Extracting large parallel corpora from movie and tv subtitles". In: Lrec 2016 (2016), pp. 923929. 
[112] Jörg Tiedemann. "Parallel Data, Tools and Interfaces in OPUS." In: Lrec. Vol. 2012. 2012, pp. 2214-2218.

[113] Guillaume Klein et al. "OpenNMT: Open-Source Toolkit for Neural Machine Translation". In: Proceedings of ACL 2017, System Demonstrations. Vancouver, Canada: Association for Computational Linguistics, July 2017, pp. 6772 .

[114] Irish Department of Health. Irish Data Protection Act 2018 (Section 36(2) Health Research) Regulations 2018. Statutory Instruments number 314 of 2018.

http : / / www . irishstatutebook . ie/eli/2018/si / 314/made / en/pdf. 2018.

[115] John R Thornbury, Dennis G Fryback, and Ward Edwards. "Likelihood ratios as a measure of the diagnostic usefulness of excretory urogram information". In: Radiology 114.3 (1975), pp. 561-565.

[116] J Martin Bland and DouglasG Altman. "Statistical methods for assessing agreement between two methods of clinical measurement". In: The lancet 327.8476 (1986), pp. 307-310.

[117] Cyrus R Mehta, Nitin Patel, and Pralay Senchaudhuri. "Exact stratified linear rank tests for ordered categorical and binary data". In: Journal of Computational and Graphical Statistics 1.1 (1992), pp. 21-40.

[118] Alan Agresti. An introduction to categorical data analysis. 2nd ed. Wiley, 2019.

[119] M Fenech, Nika Strukelj, and Olly Buston. "Ethical, social, and political challenges of artificial intelligence in health." In: Future Advocacy $\&$ Wellcome Trust, London) https://wellcome. ac. uk/sites/default/files/ai-in-health-ethicalsocial-political-challenges. pdf (accessed 26 Jul 2019) (2018).

[120] Effy Vayena, Alessandro Blasimme, and I Glenn Cohen. "Machine learning in medicine: Addressing ethical challenges". In: PLoS medicine 15.11 (2018), e1002689.

[121] Nicole Martinez-Martin, Laura B Dunn, and Laura Weiss Roberts. "Is It Ethical to Use Prognostic Estimates from Machine Learning to Treat Psychosis?" In: AMA journal of ethics 20.9 (2018), pp. 804-811.

[122] I Glenn Cohen et al. "The legal and ethical concerns that arise from using complex predictive analytics in health care". In: Health affairs 33.7 (2014), pp. 1139-1147.

[123] BlackboxNL2019. URL: https://blackboxnlp.github.io/.

[124] Sandra Wachter, Brent Mittelstadt, and Chris Russell. "Counterfactual Explanations without Opening the Black Box: Automated Decisions and the GPDR". In: Harv. JL \& Tech. 31 (2017), p. 841.

[125] Paul S Appelbaum, Charles W Lidz, and Alan Meisel. "Informed consent: Legal theory and clinical practice". In: (1987).

[126] Mason Marks. Suicide prediction technology is revolutionary. It badly needs oversight. Dec. 2018. URL: https : //www . washingtonpost . com/outlook/ suicide-prediction-technology-is-revolutionary-it-badly-needsoversight / 2018 / 12 / 20 / 214d2532 - fd6b-11e8-ad40 - cdfd0e0dd65a _ story $\cdot$ html noredirect $=$ on . 


\section{A}

\section{Appendix 1 - DeepMoji}

The following is an conversion from integer to emoji form for the DeepMoji framework.

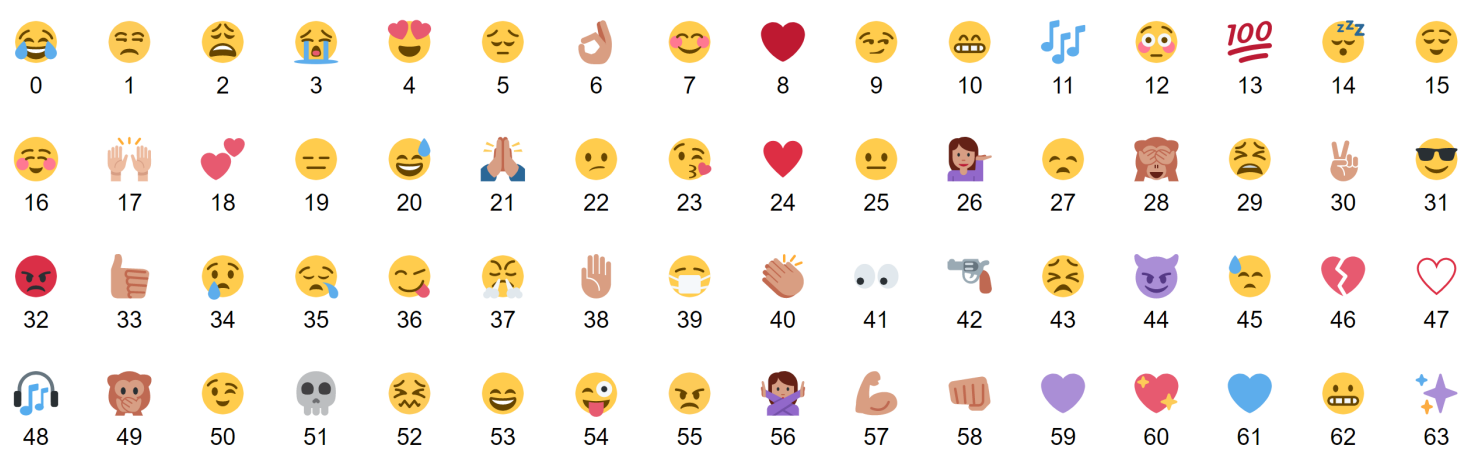

Figure A.1: Emoji representation in integer form 
A. Appendix 1 - DeepMoji 


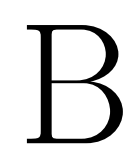

\section{Appendix 2 - Pool of Hyperparameters}

Pool of hyperparameters which were employed in the training our PHQ-4 prediction system.

- Neurons: 64, 128, 256, 512, 768, 1024

- Layers: $1,2,3,4$

- Activation functions: relu, elu, tanh, sigmoid

- Optimizer rmsprop, adam, sgd, adagrad, adadelta, adamax, nadam 


\section{C}

\section{Appendix 3 - Institutional Ethics Committee Approval}




\section{NUIG Research Ethics Committee Decision Report}

REC Application Reference Number: 19-Mar-03

Title: Passive prediction of mental health disorders

Principle Investigator: Mihael Arcan

Application Type: NEW

Meeting Date: 12 March 2019

28 March 2019

Dear Mihael,

I write to you regarding the above proposal which was submitted for Ethical review. Having reviewed your response to my letter, I am pleased to inform you that your proposal has been granted APPROVAL.

All NUI Galway Research Ethic Committee approval is given subject to the Principal Investigator submitting annual and final statements of compliance. The first statement is due on or before 27 March 2020.

See annual and final statement of compliance forms below. Section 7 of the REC's Standard Operating Procedures gives further details, and also outlines other instances where you are required to report to the REC.

Yours sincerely

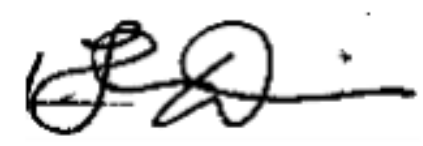

Kevin Davison

Chair, Research Ethics Committee

Figure C.1: Approved ethics application from the NUI Galway institutional ethics committee. 


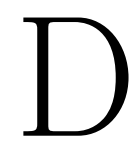

\section{Appendix 4 - Participant Information Sheet}

\section{Introduction}

Passive prediction is a new method of diagnosing mental health disorders. It is based on the concept that the way in which people talk and write can be analysed to extract certain information. In this study, we have designed a chatbot that has a normal conversation with a person. During the conversation we monitor certain aspect of the written text and use machine learning methods to predict the possible presence of depression and anxiety. The first aim of the study is to test if our machine learning methods are effective. The second aim of the study is to test if the chatbot is an effective way to interact with people.

\section{Invitation to take part in the study}

You are being invited to take part in the study to help us test our aims mentioned above. Before you decide, it is important for you to understand why the research is being done and what it will involve. This Participant Information Sheet will tell you about the purpose, risks and benefits of this research study. If you agree to take part, we will ask you to complete a consent form which is on the next page. If there is anything that you are not clear about, we will be happy to explain it to you. Please take as much time as you need to read it. You should only consent to participate in this research study when you feel that you understand what is being asked of you, and you have had enough time to think about your decision.

\section{Purpose of the study}

This study is firstly looking to test if our machine learning method is as effective in diagnosing depression and anxiety symptoms as standard questionnaires. Secondly, to test if chatbots are a suitable means to apply these machine learning modules. Anyone over 18 years of age, who can speak English can take part in this study.

4. Taking part - What it involves Do I have to take part?

It is up to you to decide whether to take part. If you do decide to take part, you will be asked to sign a consent form. If you decide to take part you are still free to withdraw at any time and without giving a reason. A decision to withdraw at any time, or a decision not to take part, will not affect your rights in any way.

What will happen to me if I take part?

Step 1) You will be asked to complete a consent form and data protection form.

Step 2) You will be asked to complete a short conversation with our chatbot about any topic you wish. We would like you to hold the conversation for at least 20 
messages, but you can leave at any time.

Step 3) You'll be asked to fill out 8 short questions. Your conversation with the chatbot, and your answers to the questionnaire is completely anonymous.

How long will this take?

We estimate no more than 10 minutes of your time, but you are free to leave at any point.

What are the benefits of taking part?

Depression and anxiety have two of the highest illness prevalence in the world, diagnosing people with these illnesses is very hard for a range of reasons. Our system aims to solve some of reasons, and hopefully help people suffering these illnesses. By taking part in this study, you in turn will help us help people. You will also have a chance to talk to a state-of-the-art artificial intelligence system.

What are the possible disadvantages and risks of taking part? While talking to our chatbot, it there is a very small chance it might say something you are not happy with. Our chatbot learns from the behaviour and language of people who talk to it, it may on occasion use language and produce apparent meaning that some consider unsuitable or inappropriate. We have done our best to train the chatbot to be nice, however during the experiment we have no control over it. If you feel uncomfortable for any reason at any point, you are free to leave. We will also recommend support services for you if you feel the need to talk to someone further. In the second half of the study we ask questions about your feelings. You might find, while you are answering it, that you would like to talk to someone about some of the issues it raises. We will be happy to recommend someone to talk to you further. You are free to skip any questions you'd rather not answer.

What happens at the end of the study?

On completion of the study you will be thanked for your participation.

Voluntary participation

Your participation in this study is voluntary, you can stop and leave at any time without any penalty. Please click the "Exit study" button to do this.

Will I receive any information after my participation?

Due to the nature of this study, you will not receive any results on the outcome of your participation in this study.

\section{Confidentially and your data rights}

By taking part in this study on passive prediction of mental health disorders, we request permission to process certain aspects of your data. As part of NUI Galway data protection policy, this study has completed an approved data protection impact statement.

In the consent form that follows this information sheet, we will ask your permission to collect and store the following data as part of our study. This data is anonymous (As per recital 26 of the GDPR), meaning we will not be able to identify any individual based on the data submitted. If you consent, we will store and process 
the following data;

- Your answer to the consent statements.

- The text inputs you provide to the chatbot.

- The answers you provide on the final questionnaire.

No additional data is recorded or stored in any way. This data will be stored for six months on NUI Galway servers. We request you do not provide the chatbot with any personal information that might be used to identify you (Name, address etc). Any indication of this will be deleted after the experiment. If you choose not to allow us collect and store this data, you will still be free to participate in the experiment, but your data will be deleted once you exit the study.

If you consent to us storing your data, you will be further asked to consent for us to process your data as follows;

- The text inputs you provided will be analysed using machine learning models. These models will predict patient health questionnaires scores based on linguistic features provided in the text.

- We will use several statistical agreement tests to compare the answers you provided on the questionnaire with the predictions from the machine learning models. This allows us to evaluate the effectiveness of these models.

Below are additional details on the data protection aspects of this study;

\section{Your data protection rights under Irish law}

The Insight Centre for Data Analytics (a Research Preforming Organisation) is a research centre within NUI Galway. You are receiving this document because Insight may hold personal information about you and as such there are rights attached to this information of which you should be aware. Depending upon the activities that Insight is performing (e.g. research, pre-trial, clinical trial, participation or attendance at events and conferences etc.) the information which Insight holds and / or process may contain personal data about you. You have rights which ensure that the information that Insight processes relating to you is factually correct, available to those authorised to access it and used for the purpose(s) stated. The list below sets out your rights as a Data Subject and they relate to the personal and/or sensitive data which Insight may process in the following formats: manual forms or documents, electronic data, photographs, video and recordings of your image or voice.

- Right to have your details used in line with data protection regulations.

- Right to information about your personal details.

- Right to access your personal details.

- Right to know if your personal details are being held.

- Right to change or remove your details.

- Right to prevent use of your personal details.

- Right to remove your details from direct marketing list.

- Right to object to the processing of your own personal data.

- Right to freedom from automated decision making.

- Right to refuse direct marketing calls or mail.

To request a copy of your own personal data please fill out the university form at the link below: https://www.nuigalway.ie/data-protection/accessandrectificationrequests/ 
In order to enable the university to process your request efficiently, please provide any details which will help us to identify you and locate your data. Please be clear about the details you are looking for if you are looking for very specific information. If you make a data access request, the university will respond within 30 days from receipt of the request. If you only want to enquire if the university holds personal data, we will respond to your request within 21 days from receipt of the request. In order to avoid unnecessary delays or confusion please be clear in your instruction that it is an enquiry and not an access request. It should be noted that under the Freedom of Information Act 1997 and 2003 records containing personal information may be released to a third party, where the public interest so requires. If you have a complaint regarding the way in which your personal data has been handled by Insight, please contact the University's data protection officer on the details below. You also have the right to make a complaint to Irish Data Protection Commissioner who can be contacted at the address below:

\section{Funding for this study}

This study has been funded by Science Foundation Ireland under grant number Insight (SFI/12/RC/2289). The funding body has no role in this study.

\section{Summary}

Thank you for reading and considering taking part in this study.

If you have any questions or concerns, or would like to know more about the study you are welcome to contact the principal investigator Email: Mihael.arcan@insightcentre.org

Relation to the data controller: Employee

Postal Address; UNLP unit, Insight Centre for Data Analytics, NUI Galway, IDA Business Park, Galway, Ireland If you have any concerns about this study and wish to contact someone independent and in confidence, you may contact 'the Chairperson of the NUI Galway Research Ethics Committee, Email: ethics@nuigalway.ie you have any concerns about the data processing aspects of this study and wish to contact someone independent and in confidence, you may contact NUI Galway data protection officer, Email: Dataprotection@nuigalway.ie 


\section{E}

\section{Appendix 5 - Participant Distress Protocol}

You have successfully left the NUI Galway study on "Passive prediction of mental health disorders". We apologize for any distress this study may have caused you. We thank you for trying the study and supporting the scientific community. If you feel the need to talk to someone about the issues that were raised in the study, or you feel the need for any level of emotional support, we encourage you to make contact with one of the following national support services:

Service Phone (From within ROI) Email

Samaritans (24 hours support) 116123 Jo@Samaritans.ie

Aware (10am to 10pm) 1800804848 Supportmail@aware.ie

Pieta House 24/7 Suicide Helpline 1800247247 


\section{$\mathrm{F}$ \\ Appendix 6 - Experimental System}

The following are screenshots from the whole experimental system. 


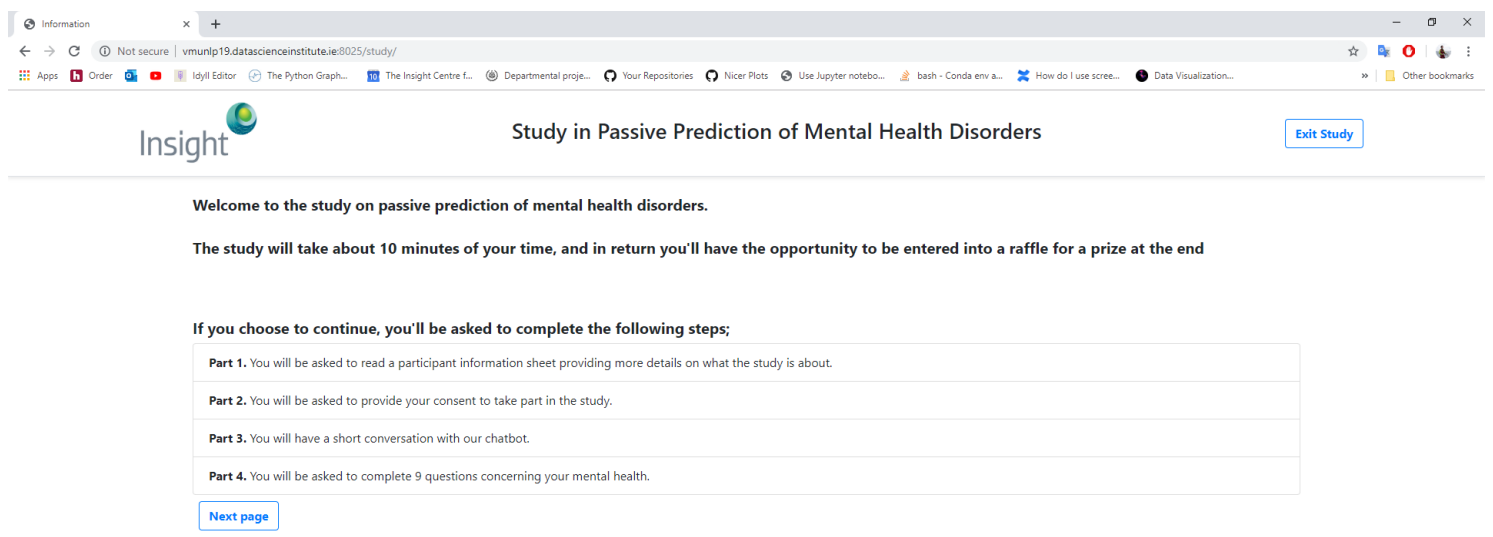

Figure F.1: Landing page

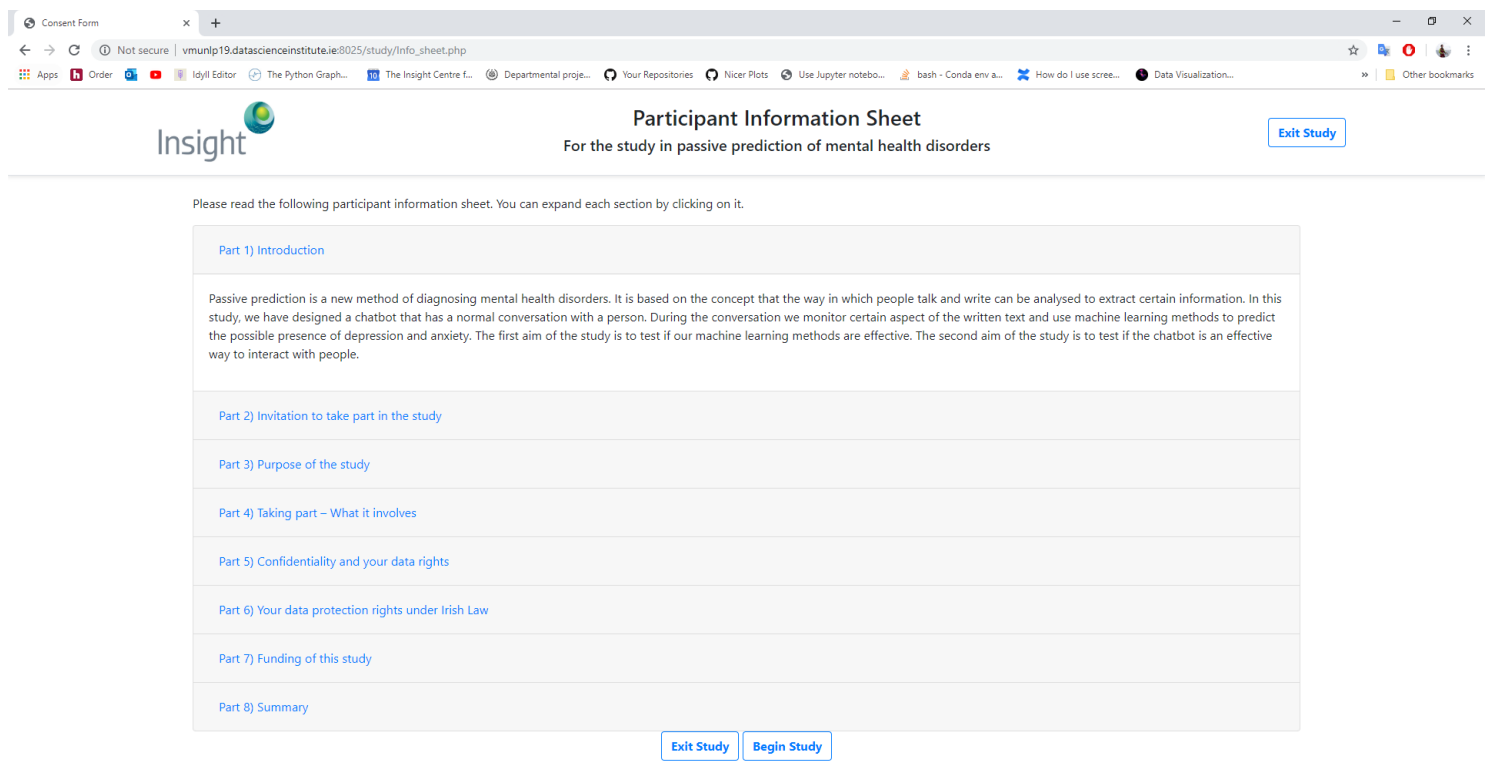

Figure F.2: Participant information page 


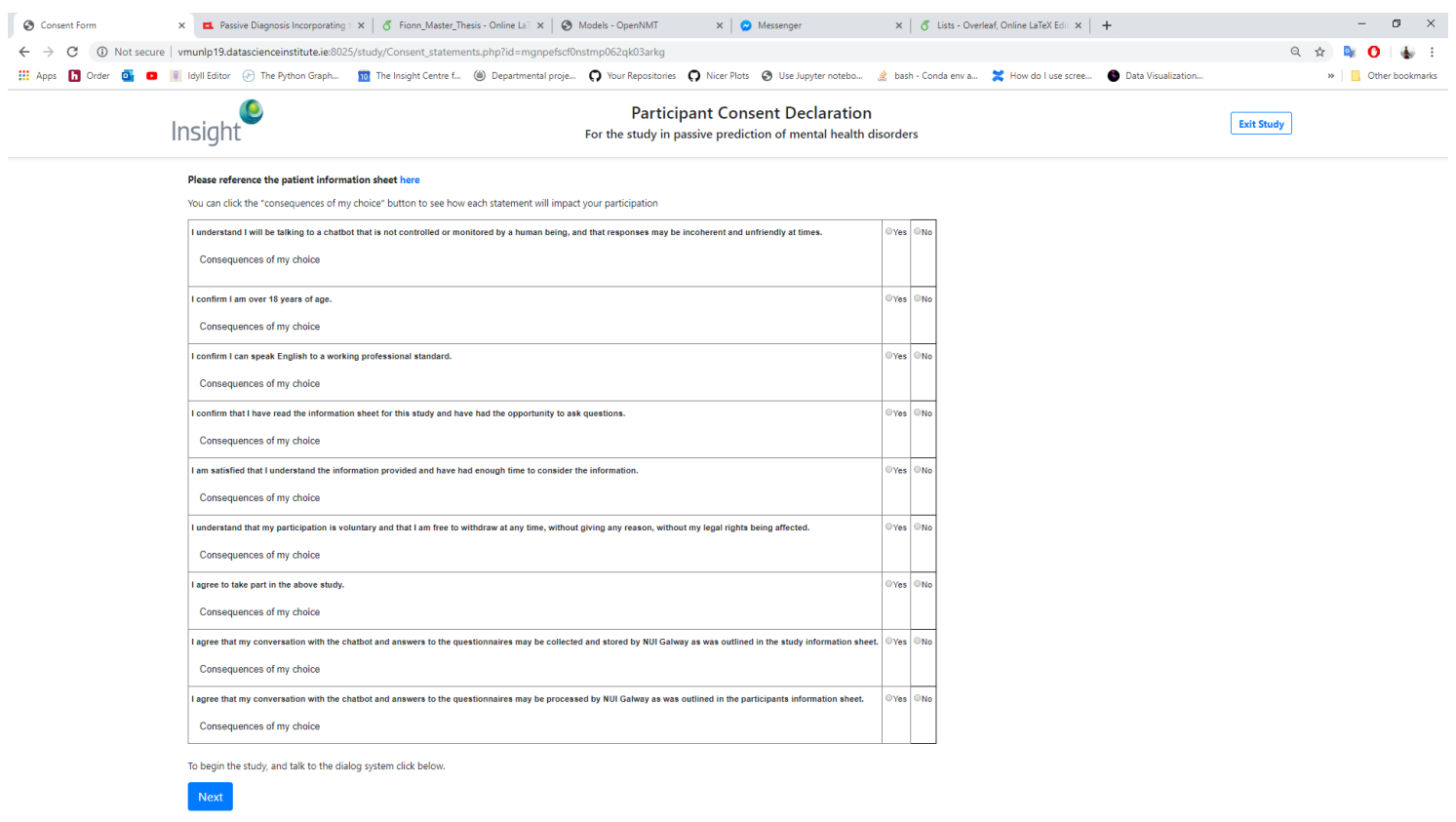

Figure F.3: Consent declaration page

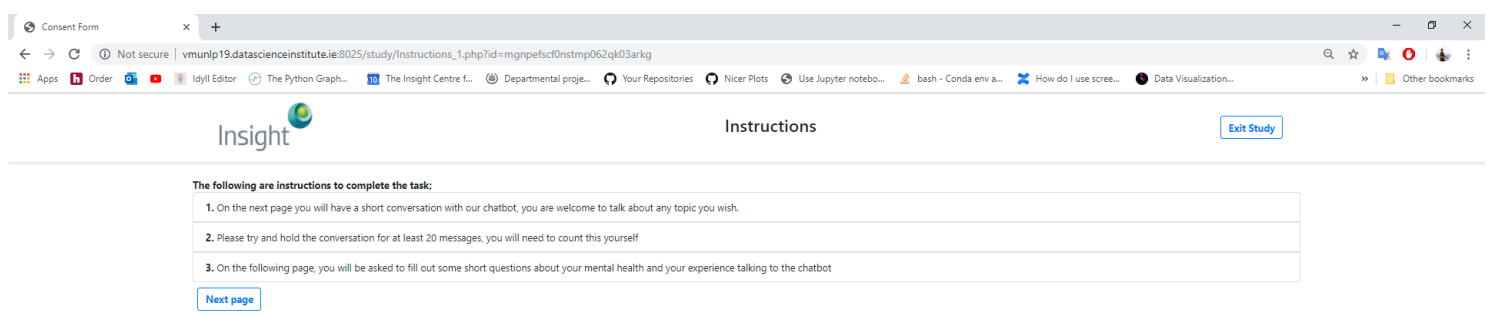

Figure F.4: Instructions reminder page 


\section{Diarvin \\ (3) Hey \\ ;:용 \\ ;: \\ y name is Marvin, nice to meet you :) \\ How are you today?}

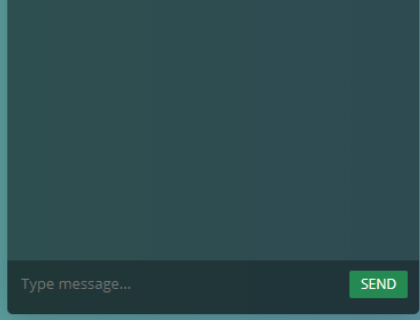

- Please try and hold the conversation for at least 20

- Yosuare welcome to talk about any topic you wish

- Sometimes Marvin can take time to think of an

answer, please be patient
- Once you're ready, clicknex

\section{\begin{tabular}{ll} 
Help Me & Next $\rightarrow$ \\
\hline
\end{tabular}}

O2017-2019, INSIGHT - National University of Ireland,
Galway.

Icons made by Icongeek26 from

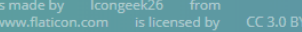

Figure F.5: Dialogue system
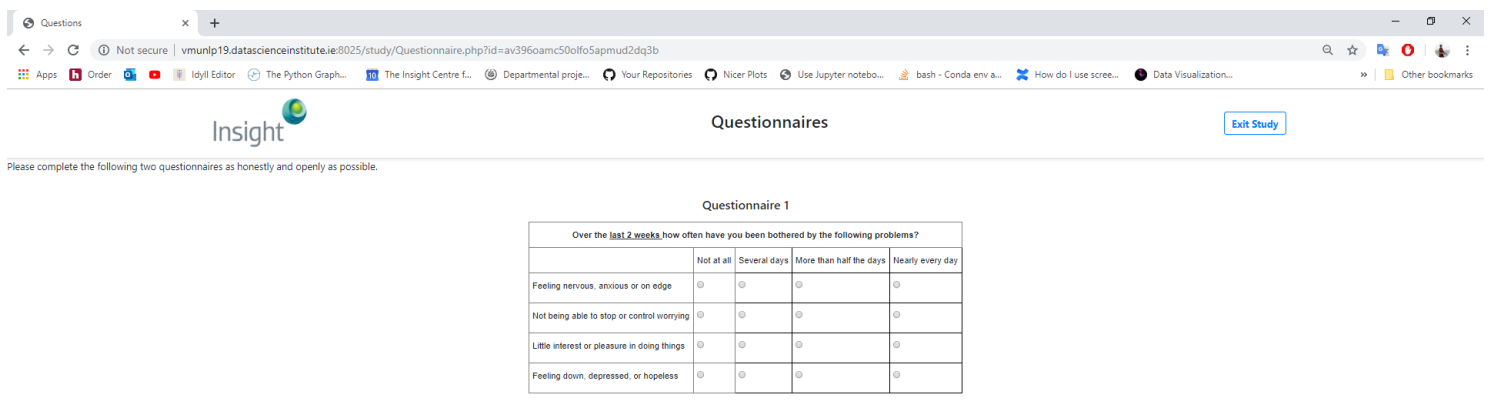

Questionnaire 2

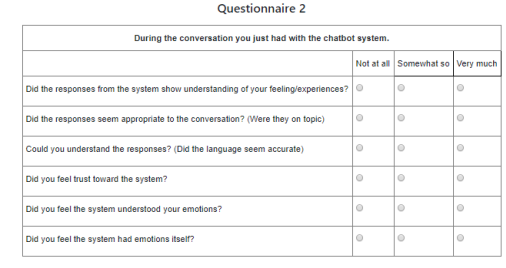

Neat

Figure F.6: PHQ and empathy questionnaire 


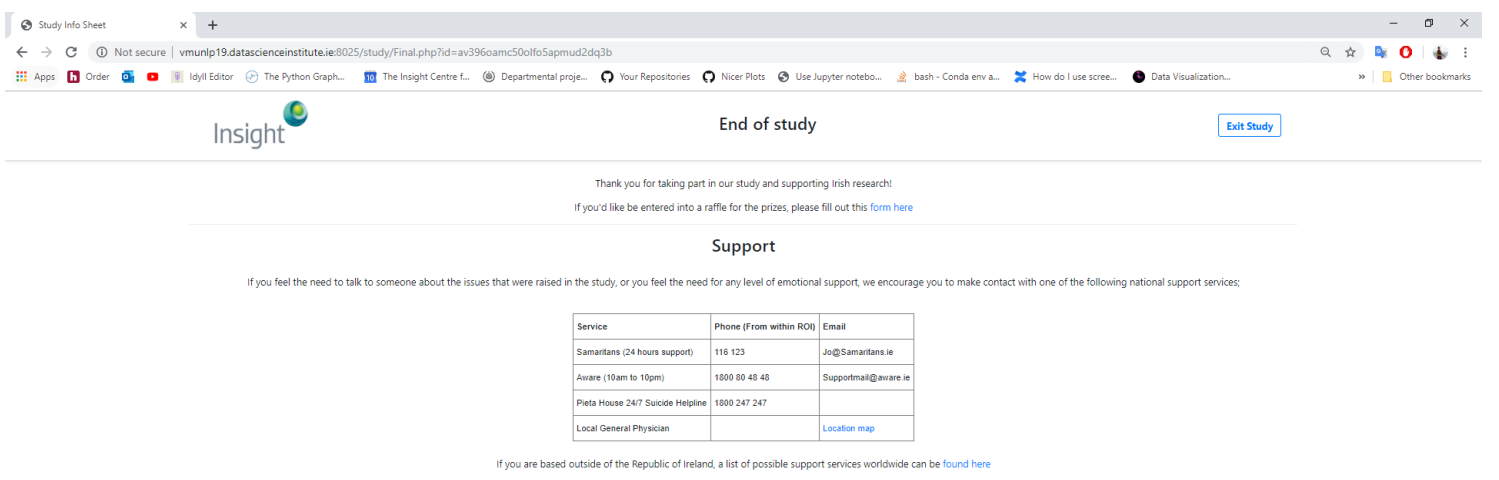

Figure F.7: Finish page 